\title{
The Versican G1 Fragment and Serum-Derived Hyaluronan-Associated Proteins Interact and Form a Complex in Granulation Tissue of Pressure Ulcers
}

\author{
Yusuke Murasawa, ${ }^{*}$ Hiroyuki Nakamura, ${ }^{\dagger}$ Ken Watanabe ${ }^{\ddagger}$ Hiroyuki Kanoh, ${ }^{\S}$ Emiko Koyama, ${ }^{\complement}$ Satoshi Fujii, Koji Kimata, ${ }^{* *}$ \\ Masahiro Zako, ${ }^{\dagger \dagger}$ Masahiko Yoneda, ${ }^{\dagger \dagger}$ and Zenzo Isogai ${ }^{{ }^{\dagger \dagger}}$

\begin{abstract}
From the Departments of Advanced Medicine, ${ }^{*}$ Bone and Joint Disease, ${ }^{\ddagger}$ and the Department of Dental Regenerative Medicine, ${ }^{\dagger}$ Center of Advanced Medicine for Dental and Oral Diseases, National Center for Geriatrics and Gerontology, Obu; the Department of Dermatology, ${ }^{\S}$ Toki Municipal Hospital, Toki; the Department of Nursing, "Kurashiki Heisei Hospital, Kurashiki; the Department of Laboratory Medicine," Asahikawa Medical University, Asahikawa; the Research Creation Support Center, ${ }^{* *}$ Aichi Medical University, Nagakute; and the School of Nursing and Health, ${ }^{\dagger \dagger}$ Aichi Prefectural University, Nagoya, Japan
\end{abstract}

Accepted for publication October 19, 2017.

Address correspondence to Zenzo Isogai, M.D., Ph.D., National Center for Geriatrics and Gerontology, 7-430, Morioka-cho, Obu, Aichi 4748511, Japan. E-mail: zenzo@ ncgg.go.jp.

\begin{abstract}
The hyaluronan ( $\mathrm{HA}$ )-rich extracellular matrix plays dynamic roles during tissue remodeling. Versican and serum-derived HA-associated protein (SHAP), corresponding to the heavy chains of inter- $\alpha$-trypsin inhibitor, are major HA-binding molecules in remodeling processes, such as wound healing. Versican G1domain fragment (VG1F) is generated by proteolysis and is present in either remodeling tissues or the mature dermis. However, the macrocomplex formation of VG1F has not been clarified. Therefore, we examined the VG1F-containing macrocomplex in pressure ulcers characterized by chronic refractory wounds. VG1F colocalized with SHAP-HA in specific regions of the granulation tissue but not with fibrillin1. A unique VG1F-SHAP-HA complex was isolated from granulation tissues using gel filtration chromatography and subsequent cesium chloride-gradient ultracentrifugation under dissociating conditions. Consistent with this molecular composition, recombinant versican $\mathrm{G} 1$, but not versican $\mathrm{G} 3$, interacted with the two heavy chains of inter- $\alpha$-trypsin inhibitor. The addition of recombinant VG1 in fibroblast cultures enhanced VG1F-SHAP-HA complex deposition in the pericellular extracellular matrix. Comparison with other VG1F-containing macrocomplexes, including dermal VG1F aggregates, versican-bound microfibrils, and intact versican, highlighted the tissue-specific organization of HA-rich extracellular matrix formation containing versican and SHAP. The VG1F-SHAP-HA complex was specifically detected in the edematous granulation tissues of human pressure ulcers and in inflamed stages in a mouse model of moist would healing, suggesting that the complex provides an HA-rich matrix suitable for inflammatory reactions. (Am J Pathol 2018, 188: 432-449; https://doi.org/10.1016/j.ajpath.2017.10.015)
\end{abstract}

Wound healing is a regulated process that occurs in four successive stages, as follows: hemostasis, inflammation, proliferation/granulation, and remodeling/maturation. During the healing of deep skin ulcers, granulation tissue transiently forms, characterized by angiogenesis and inflammatory cell infiltration; this process is essential for the subsequent remodeling process. ${ }^{1-3}$

A pressure ulcer is a common type of chronic ulcer and a refractory wound often observed in elderly patients. ${ }^{3,4}$ Although an inflammatory stage is required for wound healing, prolonged inflammation is associated with delayed wound healing, often observed in pressure ulcers. ${ }^{3}$ Moreover, these lesions exhibit various pathological features, such as angiogenesis and edema in stromal tissues, ${ }^{4}$ indicative of the variable

Supported by the Ministry of Health, Labor, and Welfare of Japan grantsin-aid H19 Chojyu-012 (Z.I., M.Y., and K.W.); the Japan Society for the Promotion of Science grant-in-aid for scientific research 25460217 (S.F. and Z.I.); the Mizutani Foundation, 2007, grant (Z.I.); and the National Center for Geriatrics and Gerontology, Japan, Research Funding for Longevity Sciences 23-15 (Z.I. and M.Y.).

Disclosures: None declared.

Current address of H.K., Department of Dermatology, Gifu University Graduate School of Medicine, Gifu, Japan. 
inflammatory conditions during the healing process. Therefore, the spatiotemporal regulation of the inflammatory response is critical for optimal wound healing in pressure ulcers.

The hyaluronan (HA)-rich extracellular matrix (ECM) is characteristically observed in remodeling tissues, such as cancer stroma and wounds. ${ }^{5-8}$ The localization of HA in the ECM is mediated by HA-binding molecules. ${ }^{9}$ Among them, versican, a large chondroitin sulfate proteoglycan, is a major HA-binding molecule in dermal connective tissue. ${ }^{10}$ In stable tissues, such as the mature dermis, versican localizes to fibrillin-microfibrils by binding fibrillin-1, which serves as a structural molecule. ${ }^{10-14}$ Localization is independent of elastic fibers in cancer and developing tissues. ${ }^{5,15-17}$ These findings suggest that versican plays tissue- or stage-specific roles through its distinct ECM formation.

Members of the a disintegrin-like and metalloproteinase with thrombospondin motif (ADAMTS) family of proteases, particularly ADAMTS-1, ADAMTS-4, ADAMTS-5, ADAMTS-9, and ADAMTS-15, cleave versican at a specific site and are, therefore, classified as versicanases. ${ }^{18}$ Versican G1 domain - containing fragments (VG1Fs) are generated by these proteases and present in mature tissues, such as the dermis and aorta. ${ }^{14,19-21}$ VG1F forms aggregates in the dermis and recruits increased levels of HA onto microfibrils through its homotypic and HA-binding activity. ${ }^{14}$ On the other hand, VG1F also induces apoptosis and angiogenesis through its biological activities in tissue remodeling and development. ${ }^{22,23}$ However, the mechanisms of these tissue-dependent VG1F functions are not fully understood.

Another HA-binding molecule, inter- $\alpha$-trypsin inhibitor $(\mathrm{I} \alpha \mathrm{I})$, covalently binds HA to generate a serum-derived HAassociated protein (SHAP)-HA complex. ${ }^{24,25}$ SHAP consists of I $\alpha$ I heavy chains (HCs) that bind HA via an ester linkage. ${ }^{25-27}$ Notably, SHAP-HA is found in the synovial fluid of patients with rheumatoid arthritis who display prolonged inflammatory reactions in their joints, ${ }^{28,29}$ the inflamed tissues of patients with inflammatory bowel disease, ${ }^{30}$ and experimental tooth infections in canines, ${ }^{31}$ indicating its critical role in inflammation.

The present study clarified the tissue-specific transient formation of a unique VG1F-SHAP-HA macrocomplex in the granulation tissue of pressure ulcers. These findings may provide new insights into the role of HA-versican-rich ECM in impaired tissue remodeling processes, such as pressure ulcers.

\section{Materials and Methods}

Antibodies, Recombinant Versican Polypeptides, I $\alpha$ Is, and SHAP-HA

Polyclonal antibodies (pAbs) against the VG1 domain, pAb 6084 and pAb 7080 (an antibody for synthetic peptide), have been described previously. ${ }^{10,14,32}$ The monoclonal antibody 2B1 against the human versican G3 (VG3) domain was acquired from Seikagaku Kogyo (Tokyo, Japan). An antibody recognizing the DPEAAE neoepitope generated by ADAMTS proteolysis of versican (pAb 8531) was characterized as almost equivalent to the commercially available antibody in our previous study.$^{14}$ Rabbit anti-mouse versican $\mathrm{pAb}$ recognizing the GAG $\beta$ domain (AB1033) was purchased from MilliporeChemicon (Temecula, CA). pAb against a synthetic peptide $\left(\mathrm{A}^{66} \mathrm{QNGN}\right.$ IKIGQDYKGR VSVP $\left.{ }^{84}\right)$ for the human versican A subdomain of human versican (V1; https://www.ncbi.nlm. nih.gov/protein; accession number NP_001157569) was generated by Operon Biotechnology (Tokyo, Japan). Its specificity was confirmed by Western blotting using conditioned media from normal dermal fibroblasts treated with chondroitinase $\mathrm{ABC}$ as an immobilized ligand ( $\mathrm{pAb}$ 10693) (Supplemental Figure S1). Rabbit anti-mouse versican polyclonal antibody against the $\mathrm{GAG} \alpha$ domain was raised against a polypeptide representing $\mathrm{D}^{380}$ to $\mathrm{E}^{957}$ of mouse versican V0 (https://www.ncbi.nlm.nih.gov/protein; accession number NP_001074718) and has been characterized previously. ${ }^{33}$ The recognition sites for pAb 6084, pAb 7080, pAb 10693, pAb 8531, AB1033, and monoclonal antibody 2B1 are shown in Figure 1. pAb 9543 (a gift from Dr. Lynn Sakai, Shriners Hospital, Portland, OR) was used as an anti-fibrillin-1 antibody. A goat pAb (clone AF2104) against tumor necrosis factor-stimulated gene-6 (TSG-6; clone AF2104) was purchased from R\&D Systems (Minneapolis, MN). A goat antibody against synthetic peptide of TSG-6 (N20, sc-21828) and a rat monoclonal antibody against TSG-6 (Q75.2.10, sc-65889) were purchased from Santa Cruz Biotechnology Inc. (Dallas, TX). A rabbit pAb against hyaluronan and proteoglycan binding link protein gene family (HAPLN)-1 (link protein, clone H93, sc-135184) and goat pAb against HAPLN-1 (K14, sc-46826; and C-14, sc46825) were purchased from Santa Cruz Biotechnology Inc. A rabbit pAb against pentraxin 3 (13797-1-AP) was purchased from Proteintech (Rosemont, IL).

A rabbit pAb against amino acid 865 to 894 of human $\mathrm{HC} 2$ of ITIH2 (LS-C165332) and a rabbit pAb against HC1 of human ITIH1 (LS-C121083) were purchased from LSBio (Seattle, WA). Mouse pAb against HC1 of human ITIH1 (10R8431) was purchased from Fitzgerald Industries International, Inc. (Acton, MA). A rabbit pAb that recognizes $\mathrm{I} \alpha \mathrm{I}$ (all HCs) was purchased from Sigma-Aldrich (St. Louis, MO; anti-I $\alpha \mathrm{I}$ antibodies). A goat pAb against bikunin (sc-21597) was purchased from Santa Cruz Biotechnology Inc. A mouse monoclonal antibody against the macrophage marker CD68 (STJ-6572) was purchased from St. John's Laboratory Ltd (London, UK). For the detection of HA, biotin-conjugated hyaluronan-binding protein (bHABP; Seikagaku Kogyo) was used. The following antibodies were used to detect antigenantibody complexes: horseradish peroxidase-conjugated anti-rabbit IgG (Dako, Glostrup, Denmark), horseradish peroxidase-conjugated anti-HIS antibody (Invitrogen, Carlsbad, CA), Alexa Fluor 633-conjugated streptavidin (Invitrogen), Alexa Fluor 568-conjugated goat anti-mouse IgG (Invitrogen), Alexa Fluor 488-conjugated goat anti-rabbit IgG (Invitrogen), and 5-nm gold particle-conjugated goat antirabbit IgG (GE Healthcare, Little Chalfont, UK). 
Recombinant versican polypeptides spanning the globular domains rVN (for VG1) and rVC (for VG3) were expressed by mammalian cells and characterized as described. ${ }^{10,14} \mathrm{I} \alpha \mathrm{I}$ was purified from bovine serum using heparin affinity chromatography, as described. ${ }^{34}$ Recombinant human HAPLN1 (2608-HP-025) and human TSG-6 (2104-TS-050) proteins were obtained from R\&D Systems. A recombinant pentraxin 3 protein was purchased from Thermo Fischer Scientific (Yokohama, Japan). To prepare the SHAP-HA complex, HA (high molecular weight; Seikagaku Kogyo) was incubated with fetal bovine serum (HyClone, South Logan, UT) and then isolated using ultracentrifugation containing cesium chloride, as described. ${ }^{24,25}$ Monomeric versican was purified from normal human fibroblast-conditioned media by using sequential anion-exchange chromatography and ultracentrifugation with cesium chloride under dissociative conditions. ${ }^{35}$

\section{Immunochemical Analysis of Wound Surface Samples and Tissue Sections}

After written informed consent was obtained from the patients, wound surface protein samples from two distinct sites of four pressure ulcers were obtained using disposable plastic tongue depressors (ORAX; Emikon, Tokyo, Japan) or cotton swabs (Nihon Menbou, Tokyo, Japan) for wound smear experiments. The samples were smeared onto glass slides (Platinum; Matsunami, Osaka, Japan), air dried for $60 \mathrm{mi}-$ nutes, fixed with $10 \%(\mathrm{v} / \mathrm{v})$ formalin (Wako, Osaka, Japan) in phosphate-buffered saline (PBS), and blocked with 50\% Starting Block T20 Blocking Buffer (Thermo Fischer Scientific) in PBS containing $0.1 \%$ Tween-20. This study was approved by the local institutional ethics committees of the National Center for Geriatrics and Gerontology (Obu, Japan).

For immunohistochemistry, formalin-fixed, paraffinembedded sections of pressure ulcers obtained at surgical excision were prepared $(n=6)$. Deparaffinized sections $(6 \mu \mathrm{m}$ thick) on glass slides were treated with $0.1 \%$ saponin for 15 minutes at $22^{\circ} \mathrm{C}$ before blocking, and the sections were blocked with blocking buffer for 60 minutes at room temperature. Then, the sections were incubated with primary antibodies in blocking buffer for 60 minutes at room temperature, washed three times in PBS containing $0.1 \%$ Tween-20 for 10 minutes at room temperature, incubated with secondary antibodies for 60 minutes at room temperature, and washed three times in PBS containing 0.1\% Tween-20 for 10 minutes. For dual immunofluorescence, tissue sections were incubated with pAb 6084, and bound antibodies were detected by using Alexa Fluor 633-conjugated anti-rabbit IgG. Probed sections were subsequently incubated with pAb 8531 preconjugated with HiLyte Fluor 555 (HiLyte Fluor 555 Labeling Kit- $\mathrm{NH}_{2}$; Dojinbo, Kumamoto, Japan) for another 60 minutes at room temperature. Sections were submerged in $20 \mu \mathrm{L}$ of Fluoromount (Diagnostic BioSystems, Pleasanton, CA), mounted with Mount-Quick (Daido Sangyo Co, Ltd, Saitama, Japan), and then sealed with a cover glass (Iwaki, Shizuoka, Japan). This part of the study was approved by the local institutional

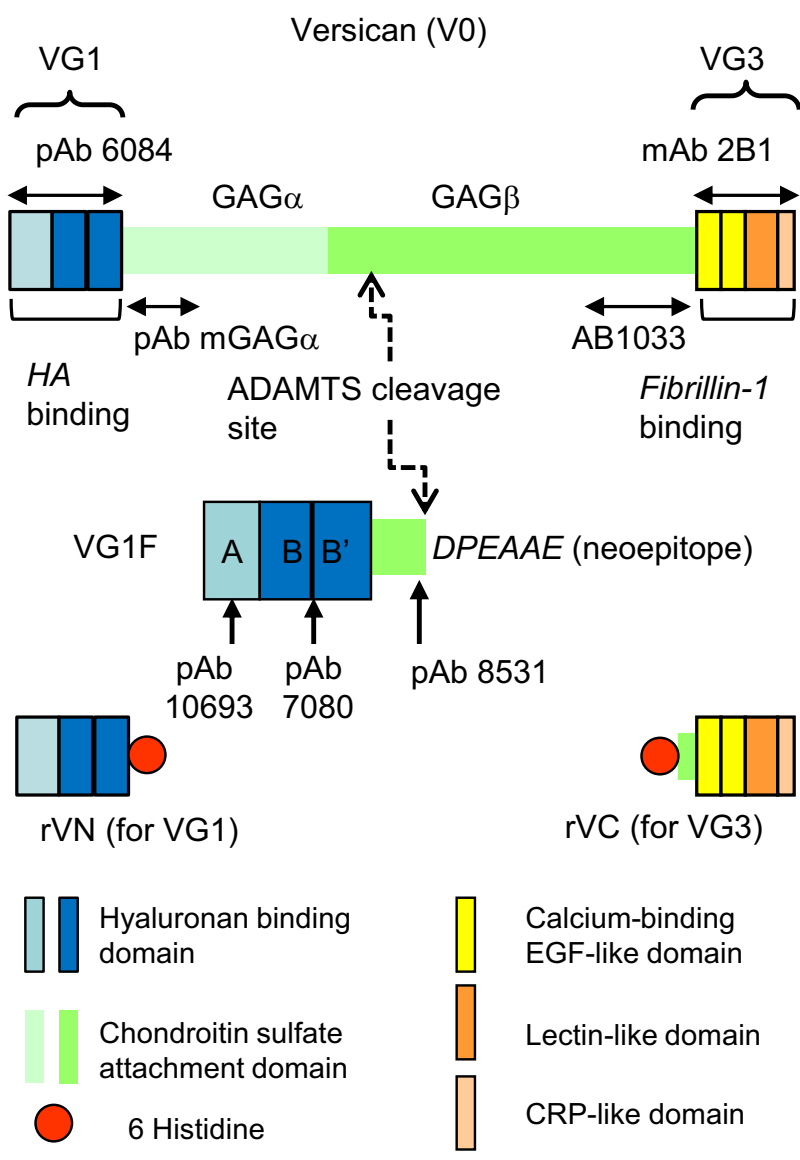

Figure 1 Schematic presentation of versican, versican G1 fragment, and antibodies used in this study. Schematic presentation of human versican (V0) and the versican G1 fragment generated by a disintegrin-like and metalloproteinase with thrombospondin motif (ADAMTS)-type versicanases. Recognition sites of anti-versican antibodies used in this study are indicated by arrows. CRP, complement regulatory protein; EGF, epidermal growth factor; mAb, monoclonal antibody; pAb, polyclonal antibody.

ethics committees of Kurashiki Heisei Hospital (Kurashiki, Japan). Tissue sections were visualized using a confocal laser microscope (Carl Zeiss LSM700 or V2URGB Stitch; Carl Zeiss, Oberkochen, Germany) that excluded nonspecific fluorescence. All processes were performed as described previously. ${ }^{14}$ Briefly, sequential dual-color images were captured, and negative controls were scanned before sample analysis. Nonspecific fluorescence was not observed in negative controls (absence of primary antibodies) using $10 \%$ laser power, 650 gain, and -0.50 offset. Subsequent samples were scanned using these standard conditions. All images were acquired using a $63 \times$ oil-immersion objective. Scanning was performed with a pinhole size of 1.0 Airy unit and eight-time line averaging. The images were stored in a $512 \times 512$-pixel, 12-bit tagged image file format.

\section{Extraction and Isolation of VG1F-Containing Aggregates}

Wound tissues were obtained from distinct pressure ulcers using protocols approved by the ethics committees of Toki 
Municipal Hospital (Toki, Japan); patients provided written informed consent $(n=2)$. Tissue samples attached to gauze for approximately 24 hours were extracted for 72 hours at $4{ }^{\circ} \mathrm{C}$ with $6 \mathrm{~mol} / \mathrm{L}$ guanidine hydrochloride $(\mathrm{GdnHCl}), 50 \mathrm{mmol} / \mathrm{L}$ Tris- $\mathrm{HCl}, 1 \mathrm{mmol} / \mathrm{L}$ phenylmethylsulfonyl fluoride, and $1 \%$ (v/v) protease inhibitor cocktail (Sigma-Aldrich), $\mathrm{pH}$ 7.5. Extracts were concentrated using an Amicon Ultra-4 centrifugal filter unit (50-kDa cutoff; Millipore, Burlington, MA) and subjected to molecular-sieve chromatography using Sepharose CL-2B (GE Healthcare) equilibrated and eluted with 4 $\mathrm{mol} / \mathrm{L} \mathrm{Gdn}$, and $50 \mathrm{mmol} / \mathrm{L}$ Tris-HCl, $\mathrm{pH} 7.5$, as described. ${ }^{14}$ For further analysis, cesium chloride was added to the void volume fractions, which were then centrifuged at $140,000 \times g$ for 48 hours at $10^{\circ} \mathrm{C}$. Protein concentrations were determined using a bicinchoninic acid protein assay kit (Pierce/Thermo Fisher, Waltham, MA) with bovine serum albumin as the standard. Dot blot analyses were performed using $5 \mu \mathrm{L}$ of sample, as described previously. ${ }^{14}$ VG1F-containing aggregates from the normal dermis were characterized in our previous study. ${ }^{14}$ Samples from the wound surfaces of separate pressure ulcers $(n=4)$ were processed using identical procedures to isolate VG1F-containing aggregates.

\section{Analysis of VG1F-Containing Aggregates}

Cesium chloride-gradient fractions containing VG1F aggregates from wounds were dialyzed three times against water at $4^{\circ} \mathrm{C}$, rotary shadowed, and observed using the electron microscope at Hanaichi Electron Microscope (Okazaki, Japan).

The fractions were spread onto glass slides (Matsunami), air dried for 60 minutes at room temperature, fixed with $10 \%(\mathrm{v} / \mathrm{v})$ formalin in PBS (Wako), and subjected to immunostaining, as described in Immunochemical Analysis of Wound Surface Samples and Tissue Sections. The slides were observed using confocal microscopy, as described above (Immunochemical Analysis of Wound Surface Samples and Tissue Sections).

For biochemical analyses, isolated samples $(10 \mu \mathrm{g})$ were treated with $0.01 \mathrm{mg} / \mathrm{mL}$ trypsin (proteomics grade, T6567; Sigma-Aldrich) in $1 \mathrm{~mL}$ of digestion buffer $(50 \mathrm{mmol} / \mathrm{L}$ $\mathrm{NH}_{4} \mathrm{HCO}_{3}, \mathrm{pH} 8.5$, and $5 \%$ acetonitrile) and $0.1 \mu \mathrm{g}$ of $\mathrm{V} 8$ protease (Sigma-Aldrich) at $37^{\circ} \mathrm{C}$ for 12 hours. ${ }^{14}$ Some of the samples were further treated with Streptomyces hyaluronidase in $50 \mathrm{mmol} / \mathrm{L}$ acetate buffer, subjected to SDSPAGE under nonreducing conditions, and then stained with Coomassie Brilliant Blue R-250, or were subjected to Western blot analysis using pAb 6084 and anti-I $\alpha \mathrm{I}$ antibodies. A blot overlay analysis using dot blots was performed, as described. ${ }^{14}$

\section{Blot Overlay Analysis and Solid-Phase Binding Assay}

To identify the ligands for VG1 in serum, a recombinant protein for VG1 $(\mathrm{rVN})^{10}$ was used in a blot overlay assay. Fetal bovine serum (JRH Bioscience, Lenexa, KS), serum-free medium secreted by normal human dermal fibroblasts, ${ }^{14}$ and purified I $\alpha \mathrm{I}$
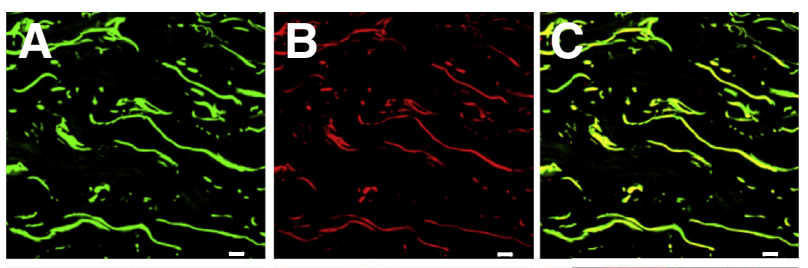

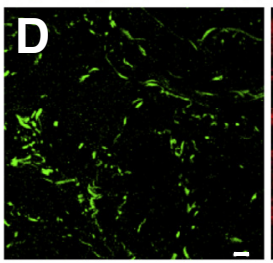

Fibrillin-1

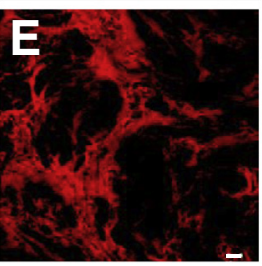

VG1

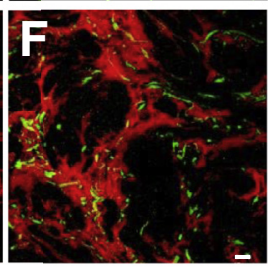

Merge
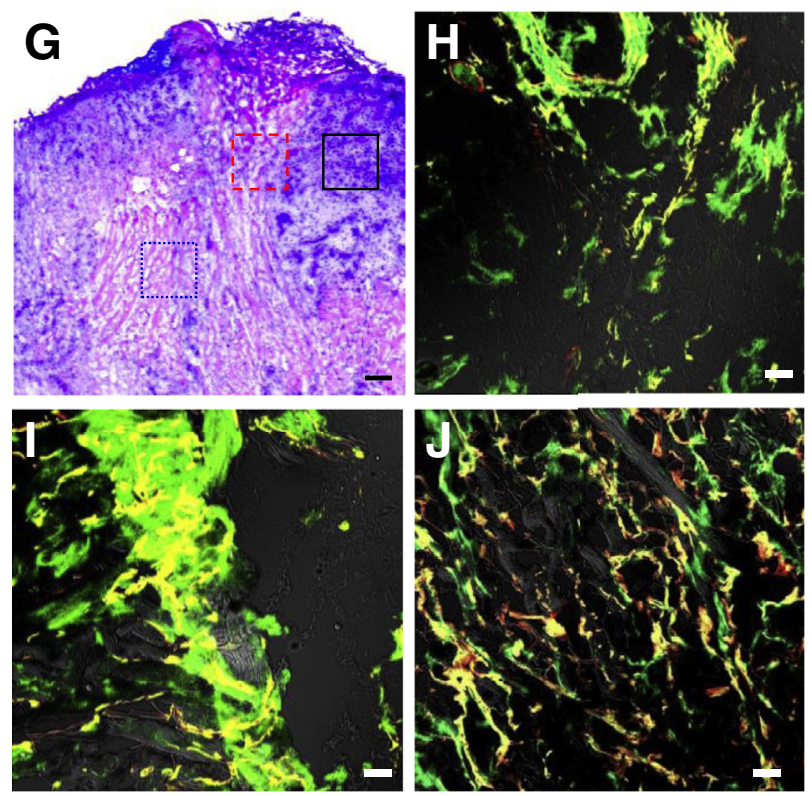

Figure 2 Localization of the versican G1 (VG1) and G3 (VG3) domains and fibrillin-1 in the dermis and granulation tissue of pressure ulcers. A-F: Immunohistochemical analysis of the normal dermis $(\mathbf{A}-\mathbf{C})$ and granulation tissue in pressure ulcers (D-F) using antibodies against fibrillin-1 [polyclonal antibody (pAb) 9543; green] and versican G1 (pAb 6084; red), as indicated. G: Pressure ulcer tissue specimens were stained with hematoxylin and eosin. Boxed areas are shown at higher magnification in $\mathbf{H}-\mathbf{J}$. H-J: Double staining with pAb 6084 (VG1; green) and monoclonal antibody 2B1 (VG3; red). H: Black boxed area in G. A lesion with granulation tissue with marked infiltration of inflammatory cells. I: Red dashed boxed area in G. Transitional lesion located between $\mathbf{H}$ and $\mathbf{J}$. J: Blue dotted boxed area in $\mathbf{G}$. A lesion with maturing connective tissue resembling the dermis. Scale bars: $10 \mu \mathrm{m}(\mathbf{A}-\mathbf{F}) ; 30 \mu \mathrm{m}(\mathbf{G}) ; 20 \mu \mathrm{m}(\mathbf{H}-\mathbf{J})$.

treated with chondroitinase ABC (Seikagaku Kogyo) were separated using SDS-PAGE under nonreducing conditions and blotted. The blots were incubated with soluble $\mathrm{rVN},{ }^{14}$ and negative controls had no rVN. Western blotting using HCspecific antibodies was also performed, as described. ${ }^{14}$

Solid-phase binding assays were performed, as described. ${ }^{14}$ Recombinant versican polypeptides $\mathrm{rVN}(0.5$ $\mu \mathrm{g} / \mathrm{mL}, 100 \mu \mathrm{L} /$ well $)$ and $\mathrm{rVC}(0.5 \mu \mathrm{g} / \mathrm{mL}, 100 \mu \mathrm{L} /$ well $)$ and bovine serum albumin $(5 \mu \mathrm{g} / \mathrm{mL}, 100 \mu \mathrm{L} / \mathrm{well}$, 


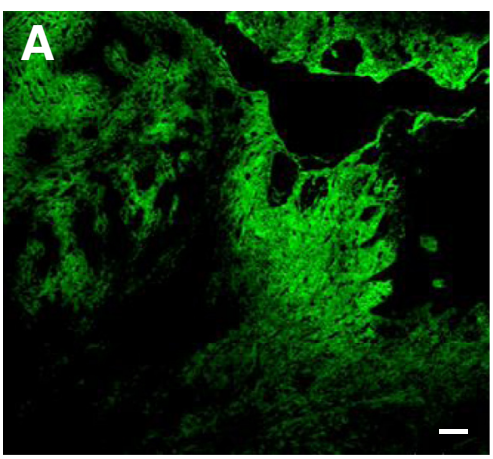

HC1

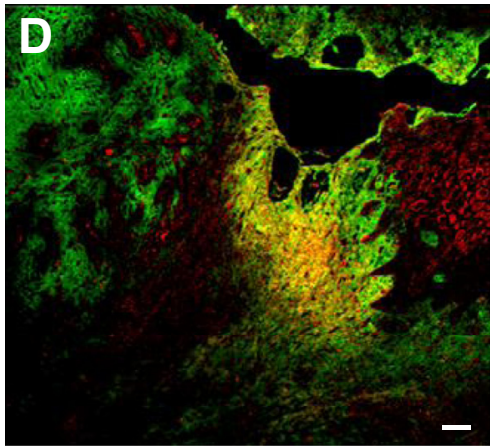

Merge (VG1-HC1)

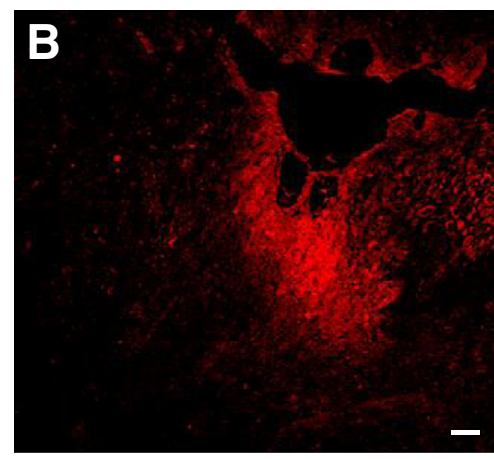

VG1

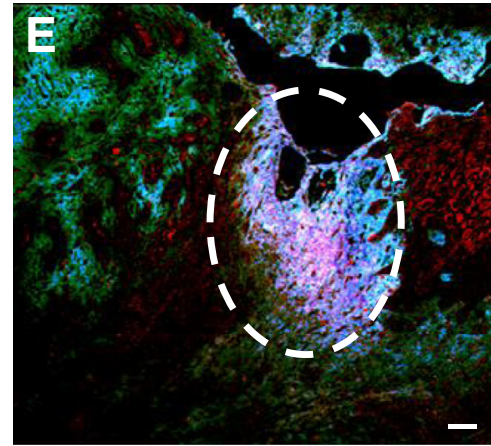

Merge (VG1-SHAP-HA)

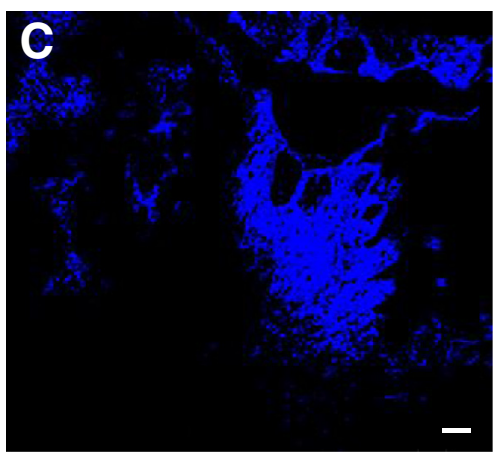

HA

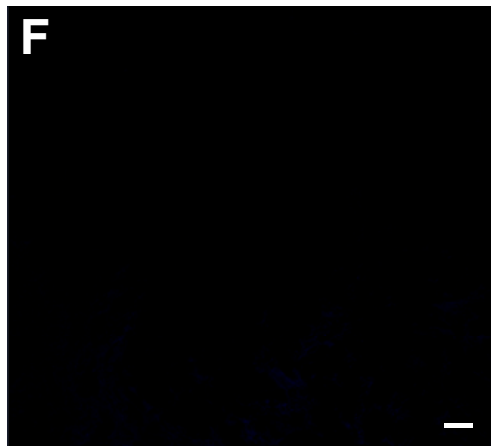

Control

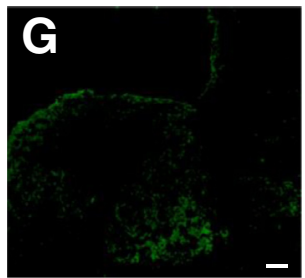

TSG-6

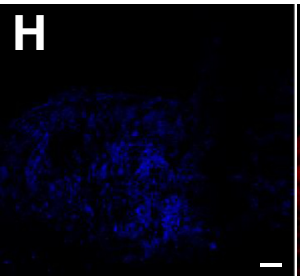

HC1

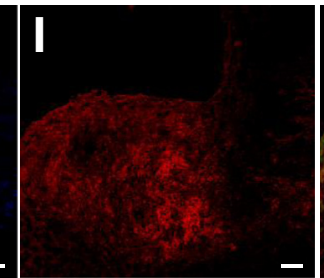

DPEAAE

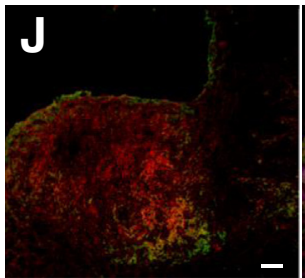

Merge

(TSG-6-DPEAAE)

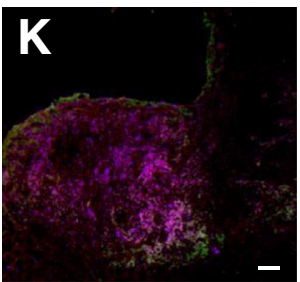

Merge

(HC1-DPEAAE)

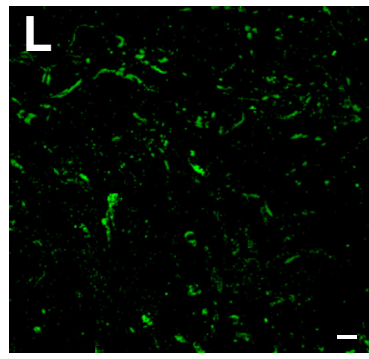

Fibrillin-1

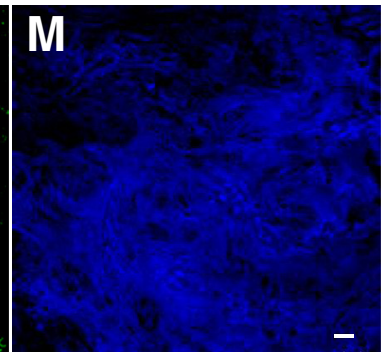

VG1

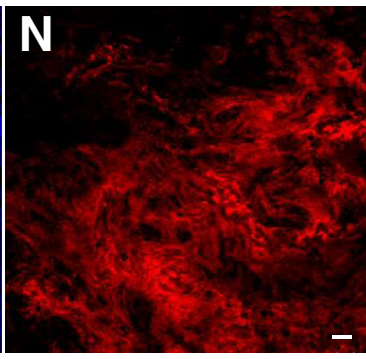

DPEAAE

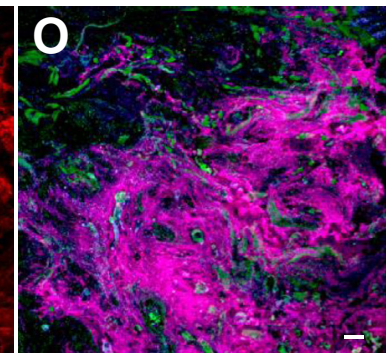

Merge

Figure 3 Localization of versican G1 (VG1) fragment, inter- $\alpha$-inhibitor heavy chains (HCs), fibrillin-1, and hyaluronan (HA) in the granulation tissue of pressure ulcers. A-F: Granulation tissue from pressure ulcers was stained with anti-ITIH1 (HC1), polyclonal antibody (pAb) 6084 (VG1), and biotin-conjugated hyaluronan-binding protein (HA) antibodies (A-E) or left unstained as a negative control (F). The area enclosed by the oval in $\mathbf{E}$ shows colocalization of VG1 and serum-derived HA-associated protein (SHAP)-HA. G-K: Granulation tissue from pressure ulcers was stained with anti-tumor necrosis factor-stimulated gene-6 (TSG-6), anti-ITIH1 (HC1), and pAb 8531 (DPEAAE neoepitope). L-0: Granulation tissues of pressure ulcers were immunostained with pAb 9543 (fibrillin-1), pAb 6084 (VG1), and pAb 8531 (DPEAAE neoepitope). L: The merged images of three antibodies are shown. The color of letters indicating molecules represents the color of the staining. Scale bars: $50 \mu \mathrm{m}(\mathbf{A}-\mathbf{F}) ; 100 \mu \mathrm{m}(\mathbf{G}-\mathbf{K}) ; 10 \mu \mathrm{m}(\mathbf{L}-\mathbf{0})$. 


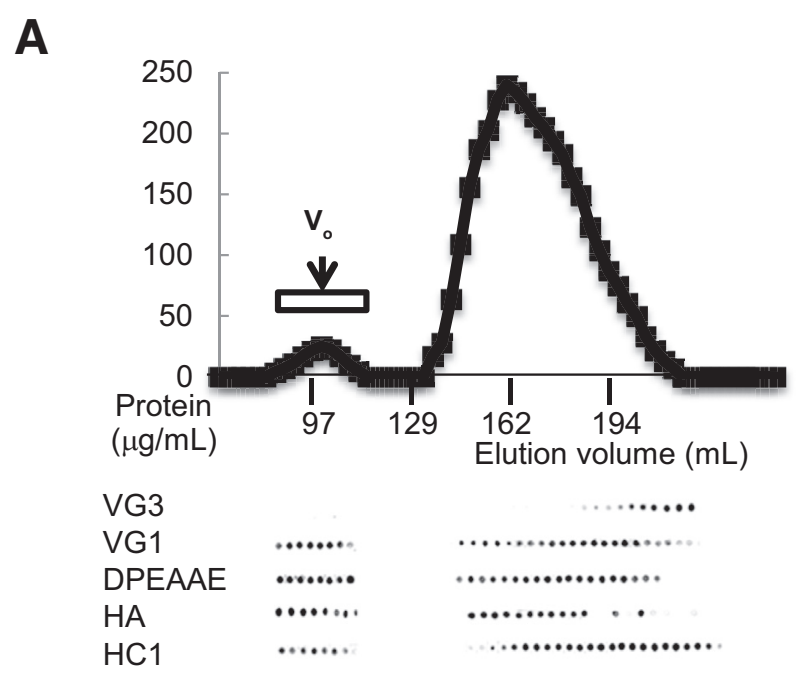

B

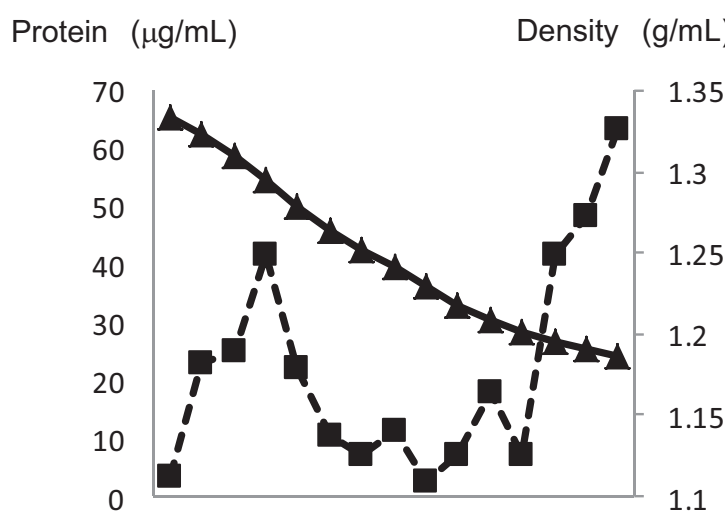

123456789101112131415

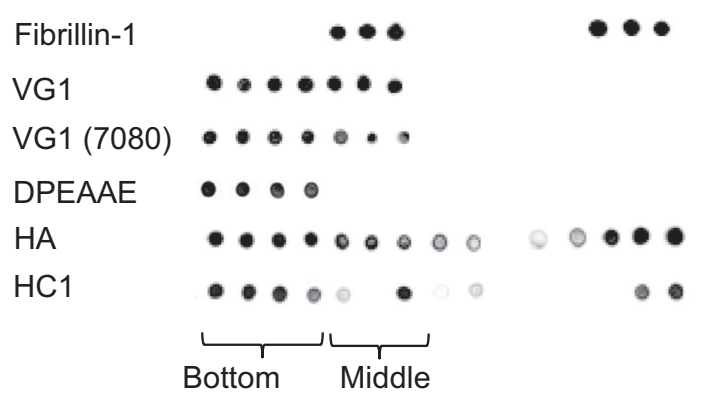

Figure 4 Isolation of versican G1 (VG1) fragment-containing aggregates from the granulation tissue of pressure ulcers. A: Representative elution profile, dot blot, and blot overlay analyses of fractions obtained by molecular-sieve chromatography. Protein extract from the granulation tissue was fractionated using CL-2B Sepharose under dissociative conditions in the presence of $4 \mathrm{~mol} / \mathrm{L}$ guanidine hydrochloride. The void volume fractions (boxed area) were further separated using cesium chloride-gradient ultracentrifugation. B: Profile of the cesium chloride density gradient ( $\rho 0=1.28 \mathrm{~g} / \mathrm{mL}$ ). VG1-containing fractions were divided into fractions 1 to 4 (bottom) and fractions 5 to 7 (middle). The density (closed triangle), protein concentration (closed square), dot blot, and blot overlay analyses are shown. Dot blots were probed with the following antibodies: polyclonal antibody (pAb) 6084 (VG1), pAb 8531 (DPEAAE neoepitope), monoclonal antibody $2 B 1$ (VG3), anti-heavy chain (HC) 1 , pAb 9543 (fibrillin-1), pAb 7080 (VG1), and anti-inter- $\alpha$-trypsin inhibitor. Blot overlay analyses using biotin-conjugated hyaluronan ( $\mathrm{HA}$ )-binding protein are also shown. $V_{0}$, position of the void volume. negative control) were coated onto multiwell plates (Sumilon, Tokyo, Japan) in carbonate buffer, $\mathrm{pH}$ 8.8. Then, they were incubated with purified $\mathrm{I} \alpha \mathrm{I}$ (threefold serial dilutions; initial dilution, $20 \mu \mathrm{g} / \mathrm{mL}$ ) in tris-buffered saline containing $2 \mathrm{mmol} / \mathrm{L} \mathrm{CaCl}_{2}$ and $0.1 \%$ bovine serum albumin and incubated for 2 hours at room temperature. Bound ligands were detected after incubation for 1 hour at room temperature with the anti-I $\alpha \mathrm{I}$ antibody diluted to $10 \mu \mathrm{g} / \mathrm{mL}$ and subsequent incubation with anti-rabbit IgG-horseradish peroxidase (Dako) diluted 1:1000 (v/v) in $2 \%$ milk/ tris-buffered saline containing $0.1 \%$ Tween-20. Antibody-antigen complexes were visualized using tetramethylbenzidine (Sigma-Aldrich), and absorbance was measured using a Benchmark Plus plate reader (Bio-Rad Laboratories Inc., Des Plaines, IL).

\section{Cell Culture Experiments}

Normal human fetal dermal fibroblasts (Lonza, Basel, Switzerland) were cultured in Dulbecco's modified Eagle's medium containing $10 \%$ fetal bovine serum at $37^{\circ} \mathrm{C}$ in a $5 \%$ $\mathrm{CO}_{2}$ incubator on glass-bottom culture dishes (Matsunami). Cells $\left(2.5 \times 10^{5}\right.$ in $250 \mu \mathrm{L}$ of medium) were seeded onto coverslips in the presence $(2 \mu \mathrm{g} / \mathrm{mL}$, final concentration) or absence of rVN. After 24 hours, $2 \mathrm{~mL}$ of media was added to the plates, and cells were cultured for an additional 24 hours. After an additional 24 hours, the cells were fixed with $10 \%$ formalin in PBS for 10 minutes at room temperature and rinsed with PBS. The fixed cells were incubated with $50 \%$ Starting Block T20 Blocking Buffer (Thermo Fischer Scientific) in tris-buffered saline containing $0.1 \%$ Tween-20 for 15 minutes at room temperature. After washing four times with PBS, slides were incubated with fluorescein isothiocyanate-conjugated anti-C-HIS antibodies (Invitrogen) for 50 minutes to detect $\mathrm{rVN}$. After washing again with PBS four times, the fixed cells were incubated in anti$\mathrm{HC} 1$ antibody for 1 hour, followed by a fluorescent secondary $\mathrm{Ab}$ for 50 minutes. To detect HA, cells were incubated in bHABP (1:500; Seikagaku Kogyo), followed by Alexa 568-conjugated streptavidin for 50 minutes each. After a final wash with PBS, coverslips were inverted onto glass slides (Matsunami), mounted with DAPIFluoromount-G (SouthernBiotech, Birmingham, AL), and then sealed using Mount Quick (Daido Sangyo, Tokyo, Japan).

The stained cells were analyzed with a scanning confocal microscope LSM 700 (Carl Zeiss), using 10\% laser power. Samples were detected with 488-, 543-, or 633-nm laser excitation and double immunofluorescence-labeled view using sequential scanning with the pinhole size set to 1.0 Airy unit and eight times line average. rVN stained with fluorescein isothiocyanate-conjugated anti-C-HIS antibodies was observed using a 488 -nm laser with 670 gain and -1 offset. $\mathrm{HC} 1$ stained with Alexa Fluor 568 antibodies was observed using a 543-nm laser with 710 gain and -1 offset. bHABP stained with Alexa Fluor 633-conjugated 
A

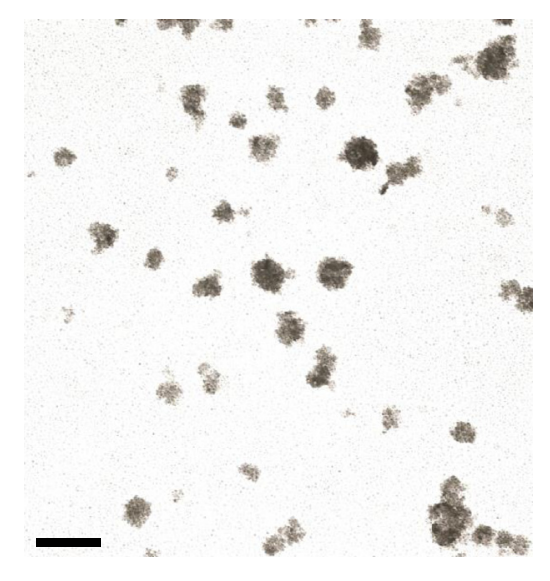

B

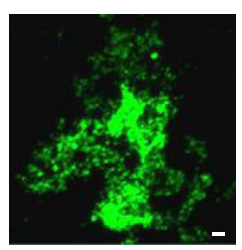

VG1

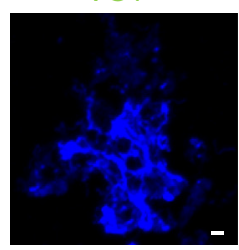

$\mathrm{HA}$

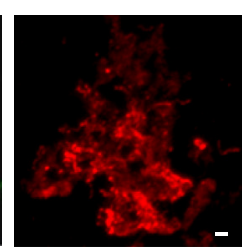

$\mathrm{HC} 1$

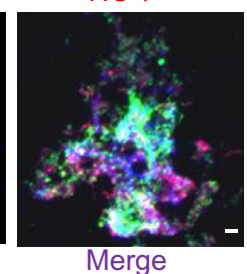

C
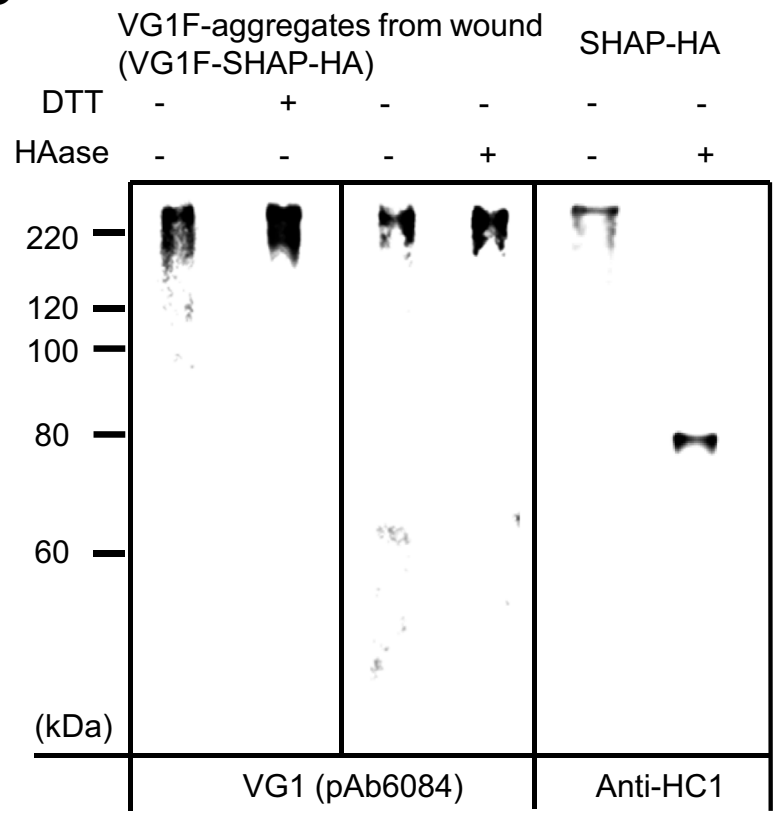

streptavidin was observed using a 633-nm laser with 800 gain and -2 offset. DAPI was observed using a $388-\mathrm{nm}$ laser with 622 gain and -10 offset. A whole-cell view was obtained using the maximum projection of nine Z-stacked images. Images were obtained using a $40 \times$ oil-immersion objective lens.

\section{Mouse Model of Moist Wound Healing}

A mouse model was established to recapitulate the wound healing process of human pressure ulcers under a moist environment. ${ }^{36}$ Three wounds were introduced on the back skin of retired ICR mice aged $>20$ weeks $(n=4$; Japan SLC, Hamamatsu, Japan) with a 6-mm trepan (Kai Industries, Seki, Japan) under appropriate anesthesia. The wounds were treated with hydrophilic ointment base (Shinsui-Nanko; Hoei, Osaka, Japan) and covered with filter paper (Whatman, 3MM Chr; GE Healthcare) that was affixed to the adjacent skin using a glue-like dressing (Ekiban-A, Taihei-Yakuhin, Japan). The ointment is effectively applied onto the wound without interference by external forces. Samples for histochemical and biochemical analyses were obtained by reexcision of the wound tissues by using a 6-mm trepan under anesthesia. The thickness of each granulation tissue was measured at biopsy. Matched tissue samples were fixed with $10 \%(\mathrm{v} / \mathrm{v})$ formalin in PBS and embedded in paraffin or used for biochemical analyses, as described in Immunochemical Analysis of Wound Surface Samples and Tissue Sections. Briefly, the obtained tissues were extracted with $6 \mathrm{~mol} / \mathrm{L}$ $\mathrm{GdnHCl}$ solution and fractionated by sieved chromatography, as described. ${ }^{14}$ This protocol was approved by the animal ethics committee of the National Center for Geriatrics and Gerontology and conducted in accordance with the Japanese guidelines for animal care.

\section{Detection of VG1F-SHAP-HA Complexes from Pressure Ulcers}

VG1F-HC-containing fragments were analyzed from a series of wound surface samples (10 pairs, total of 20 samples) obtained using cotton swabs. The samples from edematous wounds were compared with those from flat wound surfaces after the appropriate treatment for 3 to 5 weeks. The 10 paired

\footnotetext{
Figure 5 Characterization of versican G1 fragment (VG1F)-containing aggregates from pressure ulcers. A: Electron microscopic images of rotaryshadowed VG1F-containing aggregates from pressure ulcers. B: The isolated aggregates (same as $\mathbf{A}$ ) were spread onto a slide glass and incubated with polyclonal antibody 6084 (VG1), anti-heavy chain (HC) 1, and biotinconjugated hyaluronan $(\mathrm{HA})$-binding protein. Individual and merged images are shown. C: The isolated VG1F-serum-derived HA-associated protein (SHAP)-HA complex and SHAP-HA were treated with a reducing agent [dithiothreitol (DTT)] or Streptomyces hyaluronidase. Then, the samples were separated by SDS-PAGE and blotted. The blots were incubated with the antibodies shown at the bottom of the figure. The mobility of the VG1FSHAP-HA complexes is not changed by either reduction or hyaluronidase digestion. Scale bars: $100 \mathrm{~nm}$ (A); $2 \mu \mathrm{m}$ (B).
} 
samples were extracted with $6 \mathrm{~mol} / \mathrm{L} \mathrm{GdnHCl}$ solution, as described in Extraction and Isolation of VGIF-Containing Aggregates. The samples were precipitated with ethanol, and the pellets were treated with $0.01 \mathrm{mg} / \mathrm{mL}$ trypsin in 100 $\mu \mathrm{L}$ of digestion buffer $\left(50 \mathrm{mmol} / \mathrm{L} \mathrm{NH}_{4} \mathrm{HCO}_{3}, \mathrm{pH} 8.5\right.$, and $5 \%$ acetonitrile) containing $0.1 \mu \mathrm{g}$ V8 protease. The samples were resolved by $7.5 \%$ SDS-PAGE and then blotted. The blot was incubated with pAb 6084 and anti-I $\alpha$ I antibodies. Intensities of discrete signals on the blot were analyzed by calculating the number of pixels in each band, and images were quantified using ImageJ software version $1.46(\mathrm{NIH}$, Bethesda, MD; http://imagej.nih.gov/ij). The ratio of the intensity of a band positively reacted both with pAb 6084 VG1-anti-I $\alpha$ I (all HCs; Sigma-Aldrich)/total pAb 6084 (total VG1) signals was calculated. Statistical analysis was performed using the $U$-test. $P<0.05$ was considered statistically significant.

\section{Results}

\section{Distinct Localization of Versican G1 and G3 and \\ Fibrillin-1 in the Normal Dermis and Granulation Tissue}

To clarify versican-rich matrix organization in the granulation tissue of pressure ulcers and the dermis, immunohistochemical analyses were performed using characterized probes (Figure 2). The VG1 domain (including the intact versican G1 domain and the versican G1 fragment) and fibrillin-1 colocalized in the normal dermis (Figure 2, $\mathrm{A}-\mathrm{C}$ ), consistent with previous results. ${ }^{10,14}$ In contrast, colocalization of VG1 and fibrillin-1 was negligible (Figure 2, D-F), suggesting distinctive matrix organization of VG1 and fibrillin-1 in the granulation tissue of pressure ulcers. In the whole tissue of pressure ulcer (Figure 2G), predominant VG1 localization is seen in the granulation tissue (Figure $2 \mathrm{H}$ ) and the transitional region to the normal dermis (Figure 2I). Besides, VG1 and VG3 were localized similarly in the dermis-like regions of the tissue (Figure 2J). These findings showed that the versican G1 domain was located independently of the G3 domain specifically in the granulation tissue. Thus, the versican-containing matrix in the granulation tissue is different from that in the dermis.

\section{VG1F, HC, and HA Colocalization in the Granulation Tissue of Pressure Ulcers}

Because VG1 localized independently of the versican G3 domain and fibrillin-1 in granulation tissue, it is likely VG1F might assemble through its HA-binding affinity. Therefore, the localization of HA-binding molecules was examined in the granulation tissue of pressure ulcers. Routine pathological analysis for granular tissue revealed inflammatory cell infiltrate in the noncollagenous ECMs (data not shown). Within the lesion, HC1 (Figure 3A), VG1 (Figure 3B), and HA (Figure 3C) staining indicated the colocalization of $\mathrm{HC1}$ and VG1 (Figure 3D) and of VG1,
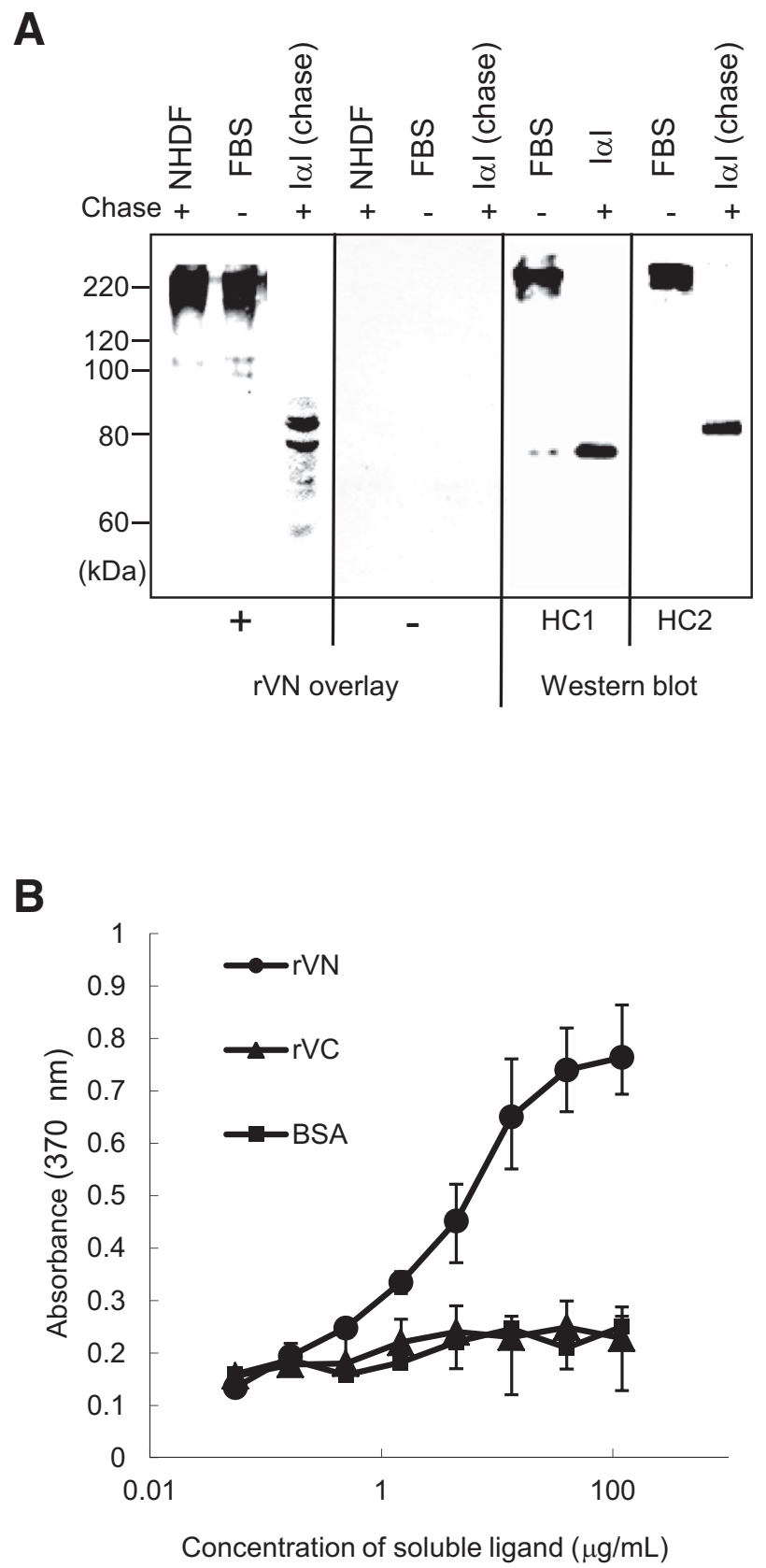

Figure 6 The versican G1 (VG1) domain interacts with inter- $\alpha$-trypsin inhibitor (I $\alpha$ I) heavy chains. A: To identify ligands for rVN (VG1 recombinant protein) in serum, blot overlay assays were performed. Conditioned media from cultures of normal human dermal fibroblasts (NHDFs), fetal bovine serum (FBS), and purified I $\alpha$ I treated with $(+)$ chondroitinase $A B C$ were used as immobilized ligands. The samples were resolved in $7.5 \%$ acrylamide gels under nonreducing conditions and blotted. For blot overlay assays, rVN (HIS-tagged recombinant VG1) was used as the soluble ligand. Bound rVN was detected by using the anti-HIS antibody. The negative control did not have the soluble ligand, rVN (indicated as rVN overlay -). Two positive signals corresponding to heavy chains (HCs) 1 and 2 in the overlay assay and Western blots probed with heavy chain-specific antibodies are shown. B: The solid-phase binding assay was performed using purified $\mathrm{I} \alpha \mathrm{I}$ as a soluble ligand. Recombinant versican globular domains, rVN, rVC (VG3 recombinant protein), and bovine serum albumin (BSA), were immobilized on the plate. Bound $\mathrm{I} \alpha \mathrm{I}$ was detected using an anti-I $\alpha \mathrm{I}$ antibody and the appropriate probes. Data are expressed as means \pm SD (B). $n=3$ independent experiments (B). 
$+r V N$
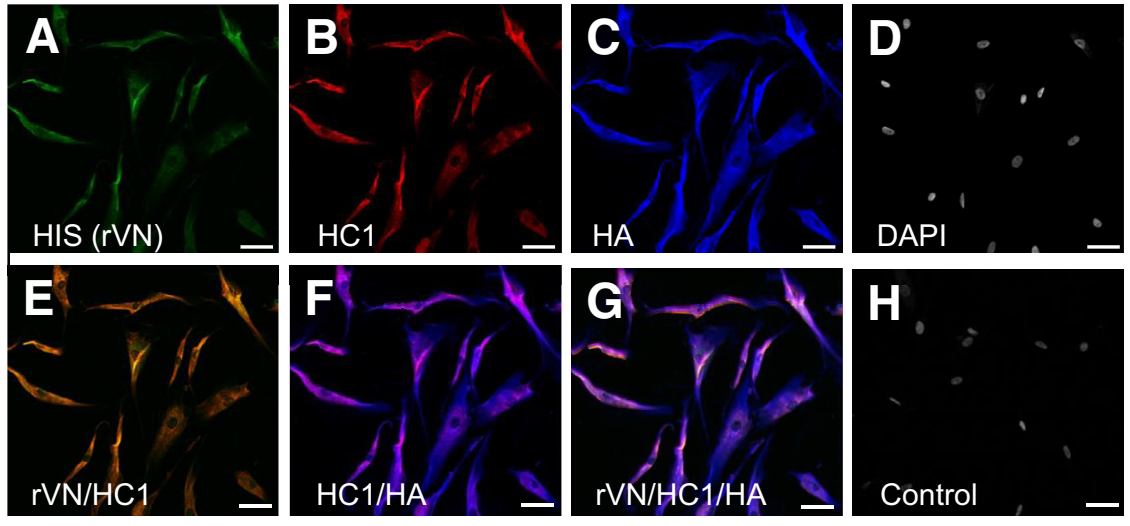

$-\mathrm{rVN}$
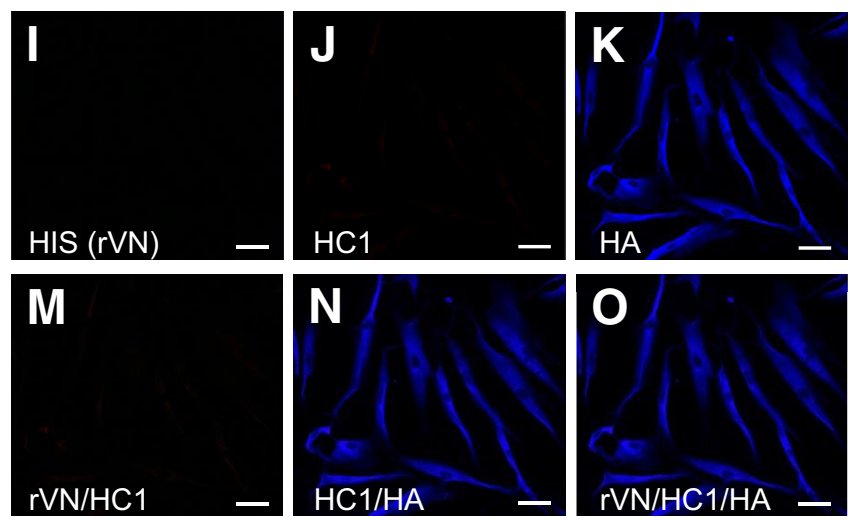

HC1, and HA (Figure 3E). Little staining was observed without primary antibodies (Figure 3F). TSG-6, another HA-binding molecule, localized in the peripheral lesion of $\mathrm{HC} 1$ and VG1F (indicated by DPEAAE antibody) (Figure 3, G-K) in the granulation tissues. Thus, TSG-6 was not localized to the lesion where $\mathrm{HC} 1$ and VG1F were colocalized (Figure 3, G-K).

Triple-immunofluorescence staining with anti-fibrillin-1 (Figure 3L), VG1 (Figure 3M), and DPEAAE neoepitope (Figure $3 \mathrm{~N}$ ) demonstrated identical localization between VG1 and the DPEAAE neoepitope (Figure 3O), but distinct localization of fibrillin-1 with VG1 or the DPEAAE neoepitope (Figure 3O). These findings indicated that most versican G1 domains were cleaved by ADAMTS-type versicanase in the granulation tissue of pressure ulcers and were localized as VG1F.

\section{Extraction and Isolation of VG1F-Containing Aggregates from Pressure Ulcer Tissues}

To identify the molecular complex containing VG1F and $\mathrm{HA}$ in pressure ulcers, tissues were extracted with $6 \mathrm{~mol} / \mathrm{L}$ $\mathrm{GdnHCl}$, and the extracts were subjected to gel filtration in the dissociative condition. Notably, VG1 detected by $\mathrm{pAb}$ 6084 was detected in fractions eluted in the void volume
Figure 7 Exogenous versican G1 (VG1) fragment induces serum-derived hyaluronan-associated protein-hyaluronan (SHAP-HA) deposition into the pericellular matrix of dermal fibroblasts. Normal human dermal fibroblasts were cultured with the presence $(\mathbf{A}-\mathbf{H})$ or absence $(\mathbf{I}-\mathbf{P})$ of exogenous rVN (HIS-tagged recombinant VG1) for 48 hours. A and I: The added rVN (green) was detected by using anti-HIS antibody. B, C, J, and K: Heavy chain ( $\mathrm{HC}) 1$ (B and $\mathbf{J}$ ) and $\mathrm{HA}$ ( $\mathbf{C}$ and $\mathbf{K}$ ) were detected using anti-HC1 and biotin-conjugated $\mathrm{HA}$ -binding protein, respectively. D and L: DAPI staining (blue) was used to visualize the nucleus. $\mathbf{E}$ - G and $\mathbf{M}-\mathbf{0}$ : Merged images for rVN, HC1, and HA are shown, as indicated. $\mathbf{H}$ and $\mathbf{P}$ : Negative controls omitting primary probes are shown. Representative results from three independent experiments are shown. Scale bars $=50 \mu \mathrm{m}(\mathbf{A}-\mathbf{P})$.

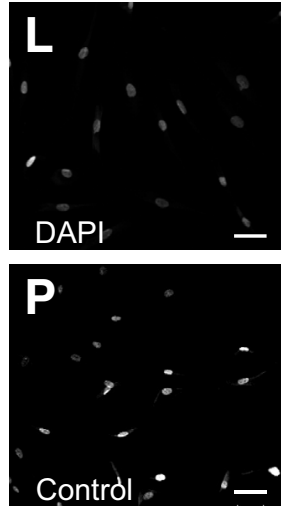

(Figure 4A). The void-volume fractions also reacted with anti-DPEAAE, pAb 7080 (anti-VG1), anti-heavy chain antibodies, and bHABP. However, they were faintly positive for anti-VG3 (Figure 4A). These results indicated the presence of VG1F-containing aggregates in the fractions.

For further characterization of the macromolecules, the void-volume fractions (Figure 4A) were subjected to cesium chloride ultracentrifugation under dissociating conditions (Figure 4B). VG1-containing aggregates were collected in seven fractions (Figure 4B), designated as bottom (1 to 4 ) or middle (5 to 7 ) fractions. Bottom fractions reacted with anti-VG1, anti-DPEAAE, and anti-HC1 antibodies, but not with antibodies against fibrillin-1 (Figure 4B); the middle fractions reacted with anti-VG1 and anti-fibrillin-1. Consistent with these data, the middle fractions did not react with anti-DPEAAE antibody (Figure 4B), suggesting that the VG1 domain was not cleaved by ADAMTS-type versicanases. VG1F-containing aggregates in other preparations from different wound tissues were isolated using the same experimental procedures and showed similar biochemical properties. VG1Fcontaining aggregates were prepared similarly from separate wound surface samples (data not shown). These results were consistent with the immunohistochemical findings that VG1F, HCs, and HA colocalized to the surface layer of 
A

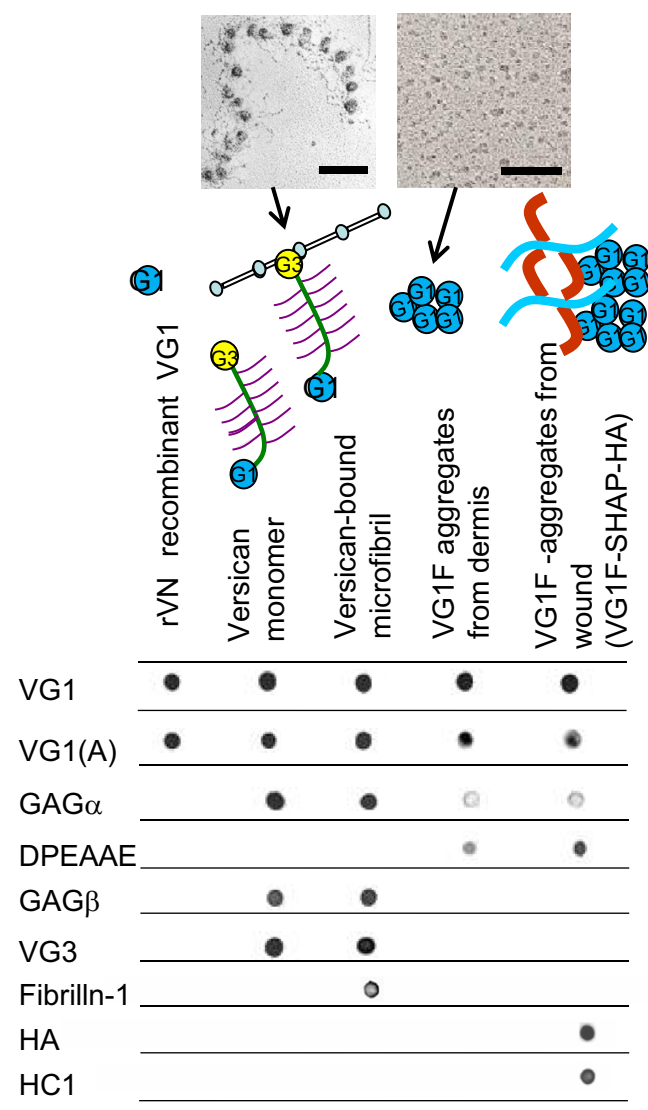

B

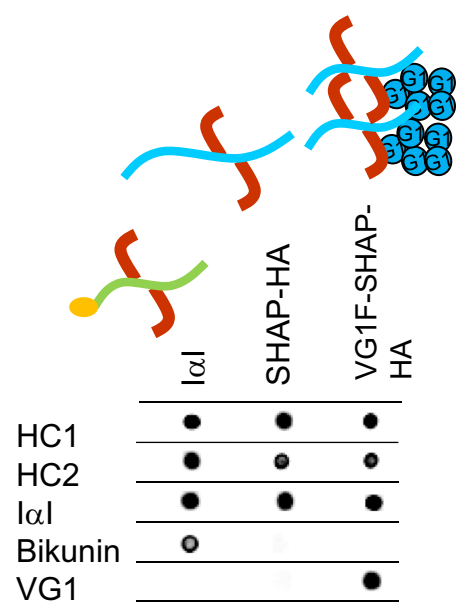

C

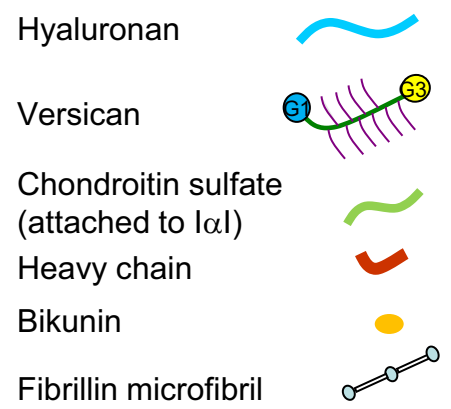

the wound tissue (Figure 3). Together, these findings suggested distinct biochemical properties of VG1F-containing aggregates of pressure ulcers.

\section{Characterization of VG1F-Containing Aggregates from Granulation Tissue}

The morphological and biochemical properties of VG1Fcontaining aggregates were next characterized from the granulation tissue of pressure ulcers. Electron microscopic analysis showed round aggregates ( 25 to $50 \mathrm{~nm}$ in diameter) with an irregular, electron-dense periphery (Figure 5A). When the aggregates were spread onto the glass and observed at light microscopy level using immunofluorescence, VG1F, $\mathrm{HC} 1$, and HA were found to be colocalized (Figure 5B).

To further analyze the aggregates, samples were treated with proteases and subjected to SDS-PAGE and immunoblotting; however, the selected treatments, including reduction (Figure 5C) and trypsin digestion (Supplemental Figure S2), were unable to generate fragments small enough to enter the gel, suggesting cross-linking among the composed molecules. Furthermore, hyaluronidase treatment did not alter the mobility of the high-molecular-weight aggregates detected with anti-VG1 (Figure 5C), whereas hyaluronidase-treated SHAP-HA exhibited altered mobility by Western blot with anti-HC1, as expected (Figure 5C). Even when the samples were treated with trypsin alone or trypsin and V8 protease, most of the aggregates were resistant to protease treatments (Supplemental Figure S2). Nevertheless, Western blot analysis with pAb 6084 (antiVG1) or staining of the resultant fragments with Coomassie Brilliant Blue R-250 displayed similar patterns (Supplemental Figure S2). However, these analyses may not

Figure 8 Comparison of the versican G1 fragment-serum-derived hyaluronan-associated protein-hyaluronan (VG1F-SHAP-HA) complex with other VG1 or inter- $\alpha$-trypsin inhibitor (I $\alpha \mathrm{I})$-heavy chain $(\mathrm{HC})$-containing molecules or complexes. Dot blot and blot overlay assays were performed using various probes. A: Versican-containing molecules [including rVN, full-length versican monomer purified from conditioned medium, versicanbound microfibrils, VG1F aggregates from the normal dermis, and VG1Fcontaining aggregates from wounds (VG1F-SHAP-HA)] were spotted onto membranes. The blots were incubated with antibodies or ligands, as indicated, and polyclonal antibody (pAb) 6084 (VG1) reactivity was used as a reference for the immobilized ligands. Dot blots were probed with the antibodies, as follows: pAb 6084, pAb 10693 (antibody for A subdomain [VG1(A)]), pAb anti-mouse GAG $\alpha$ (GAG $\alpha$ domain), pAb 8531 (DPEAAE neoepitope), AB1033 (GAG $\beta$ domain), monoclonal antibody 2B1 (VG3), pAb 9543 (fibrillin-1), and anti-HC1. Blot overlay analyses using biotin-conjugated HA-binding protein are also shown. Rotary-shadowed-electron microscopic images of versican-bound microfibrils and VG1F aggregates from the normal dermis are shown at the top. Schematic illustrations of the molecular composition of each complex are indicated. B: I $\alpha \mathrm{I}, \mathrm{SHAP}-\mathrm{HA}$, and VG1F-containing aggregates from wound were spotted onto membranes, which were probed with antibodies or binding ligands. Anti-HC1 antibody reactivity was used as a reference for the immobilized ligands. Dot blots were probed with the following antibodies: anti-HC1, anti-HC2, anti-I $\alpha$ I, anti-bikunin, and pAb 6084. Schematic illustrations of the molecular composition of each complex are presented at the top. C: The legend of the symbols. Scale bars $=100 \mathrm{~nm}(\mathbf{A})$. 
A

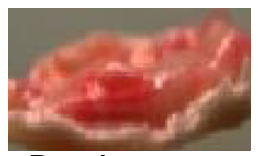

Day 1

C
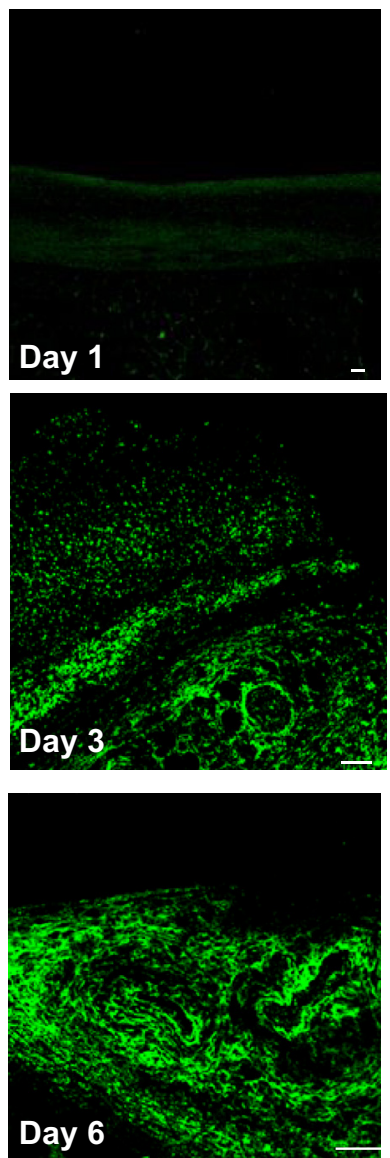

HA

D

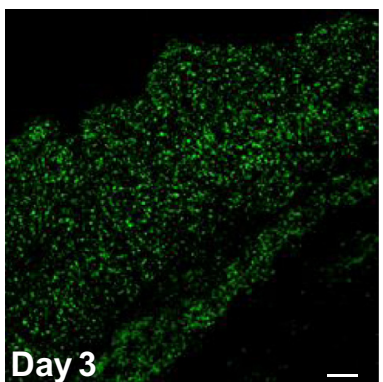

HA

Day 3
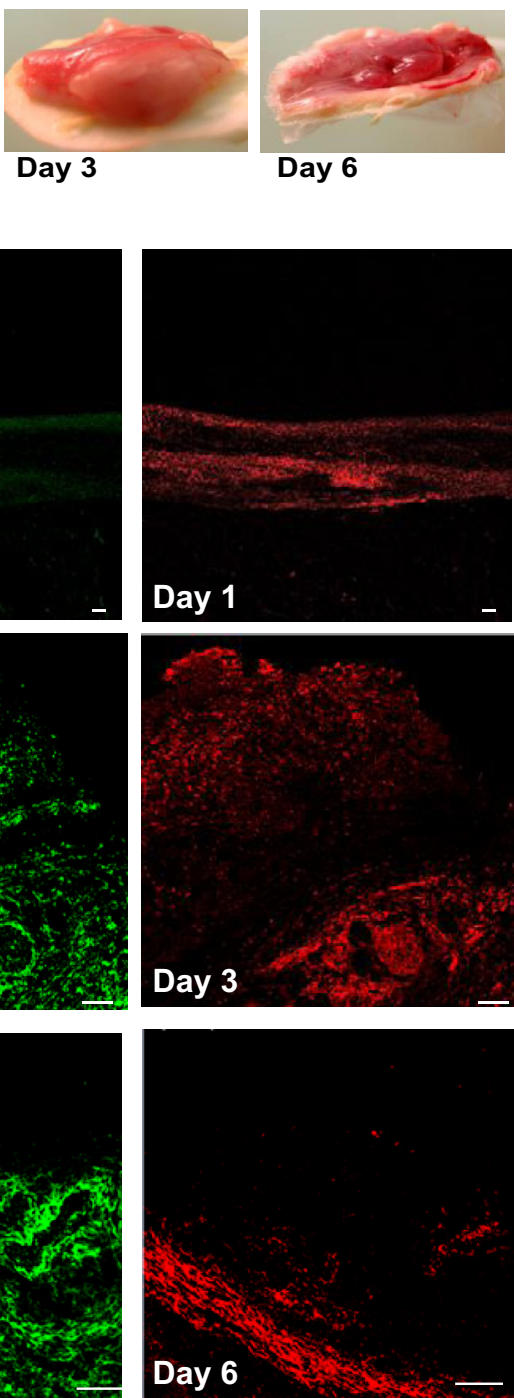

DPEAAE

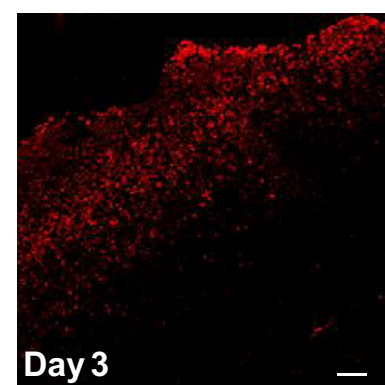

DPEAAE
B
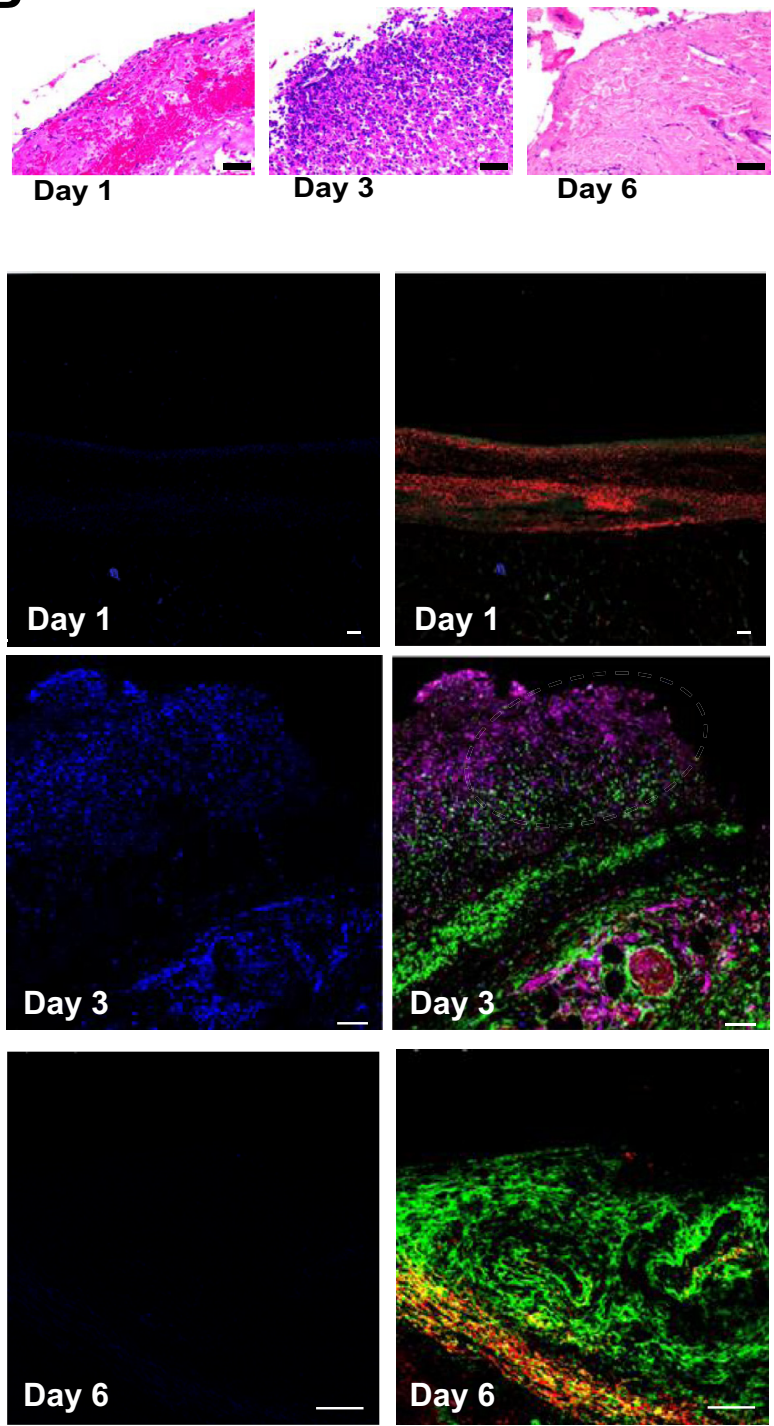

$\mathrm{HC} 1$

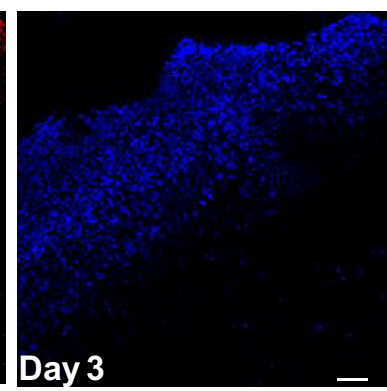

Macrophage

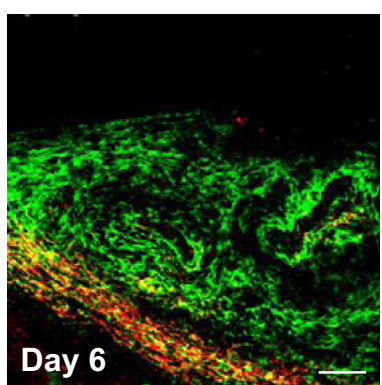

Merge

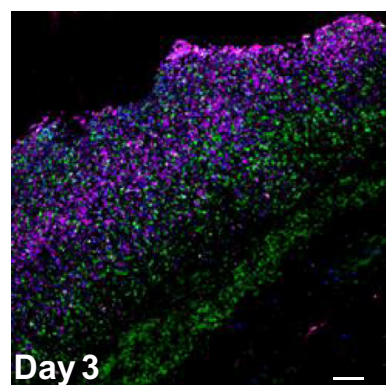

Merge

Figure 9 Transient versican G1 fragment-serum-derived hyaluronan-associated protein-hyaluronan (VG1F-SHAP-HA) complex formation in a mouse model of moist wound healing. A: Representative images from wound tissues from the moist wound-healing model are shown. Edematous granulation tissues were observed at day 3 after wounding; however, the granulation tissues were nonedematous and thin at days 1 and 6 . The thickness of the granulation tissues is as follows: day 1, $0.77 \pm 0.10 \mathrm{~mm}$; day 3, $2.55 \pm 0.17 \mathrm{~mm}$; and day 6, $0.82 \pm 0.04 \mathrm{~mm}$. B: Tissue specimens were stained with hematoxylin and eosin. C: Isolated tissues were stained by using the indicated probes. The VG1F-SHAP-HA complex was observed on day 3 in specific lesions, but was absent at days 1 and 6. The color of letters indicating molecules represents the color of the stain. The merged images indicate the VG1F-SHAP-HA complex. D: Immunohistochemical analysis of wound tissue taken from day 3. The specimen was stained with the indicated probes, and the merged images show VG1F colocalization, as detected by probes for HA, DPEAAE epitope, and macrophages (CD68). The merged images show macrophage/complex colocalization. Data are expressed as means \pm SD (A). $n=4$ independent experiments (A). Scale bars $=50 \mu \mathrm{m}(\mathbf{B}-\mathbf{D})$. HC, heavy chain. 

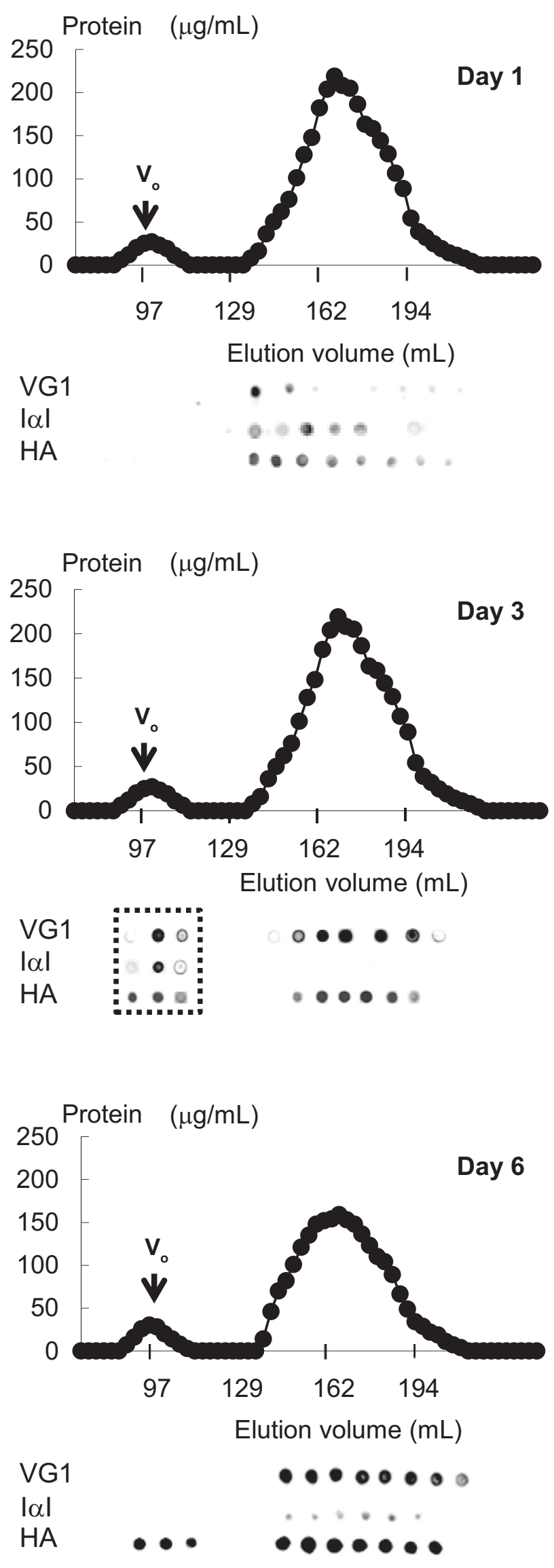

exclude the presence of the other molecules in the aggregates because the aggregates were resistant to protease treatment. Because the aggregates were isolated from a chronic wound, which is known to exhibit prominent proteolytic activity, ${ }^{37,38}$ we hypothesized that heavily cross-linked VG1F, HCs, and HA are present in the aggregates. To exclude the presence of the other candidate HA-binding molecules in the VG1F-containing aggregates, a series of antibodies for TSG-6, HAPLN1, and pentraxin 3 were tested. However, none of these HA-related molecules was present in the aggregates (Supplemental Figure S3).

\section{Interaction between $\mathrm{HCs}$ and the Versican G1 Domain}

Because the VG1F-SHAP-HA complex was detected in the granulation tissue of pressure ulcers, where serum and ECM molecules were merged together, the interactions between VG1F and HCs of SHAP were examined. A blot overlay assay using fetal bovine serum as the immobilized ligand showed that $\mathrm{rVN}$ (a recombinant protein for VG1) selectively bound an approximately $230-\mathrm{kDa}$ molecule, later identified as I $\alpha$ I by immunoblotting with anti-HC1 and anti-HC2 antibodies (Figure 6A). When the primary ligand, rVN, was omitted, no signal was observed (Figure 6A).

To determine the I $\alpha$ I heavy chains that bound VG1F, purified $\mathrm{I} \alpha \mathrm{I}$ treated with chondroitinase $\mathrm{ABC}$ was used as an immobilized ligand for blot overlay analyses and immunoblotting. The results revealed that $\mathrm{rVN}$ bound to $\mathrm{HC} 1$ and HC2 (Figure 6A). Moreover, soluble I $\alpha \mathrm{I}$ bound to immobilized VG1 (rVN), but not VG3 (rVC; a recombinant protein for VG3), in a solid-phase binding assay (Figure 6B). Thus, these data indicate that $\mathrm{I} \alpha \mathrm{I}$ interacts with VG1, consistent with the composition of the VG1F-SHAPHA complex isolated from pressure ulcers.

VG1F Enhances VG1F-SHAP-HA Complex Deposition in the Pericellular Matrix Produced by Dermal Fibroblasts

The effects of VG1F on SHAP-HA complex deposition were tested using a dermal fibroblast culture in the presence of serum. Notably, rVN was incorporated into the ECM in the presence of $\mathrm{rVN}$ (Figure 7, A and I), whereas the addition of $\mathrm{rVN}$ enhanced the deposition of $\mathrm{HC} 1$ (Figure 7, B and $\mathrm{J}$ ) and HA (Figure 7, $\mathrm{C}$ and $\mathrm{K}$ ) into the pericellular ECM, but not the nucleus (Figure 7, D and L).

Figure 10 Biochemical characterization of versican G1 fragment-serum-derived hyaluronan-associated protein-hyaluronan (VG1F-SHAP-HA) complex formation in a mouse model of moist wound healing. Representative elution profile, dot blot, and blot overlay analyses of fractions obtained by molecular-sieve chromatography. The extracts from wound tissue at days 1,3 , and 6 were fractionated using CL-2B Sepharose in the presence of $4 \mathrm{~mol} / \mathrm{L} \mathrm{GdnHCl}$. Dot blots were probed with the following antibodies: polyclonal antibody 6084 (VG1) and anti-inter- $\alpha$-trypsin inhibitor ( $\mathrm{I} \alpha$ ). A blot overlay analysis with biotin-conjugated HA-binding protein is also shown. The area enclosed by the dotted box shows the presence of the VG1F-SHAP-HA complex. $V_{0}$, position of the void volume. 
A

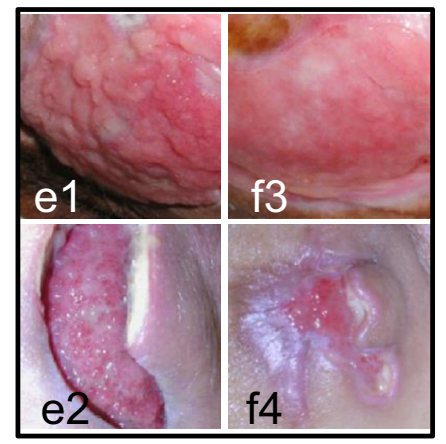

D
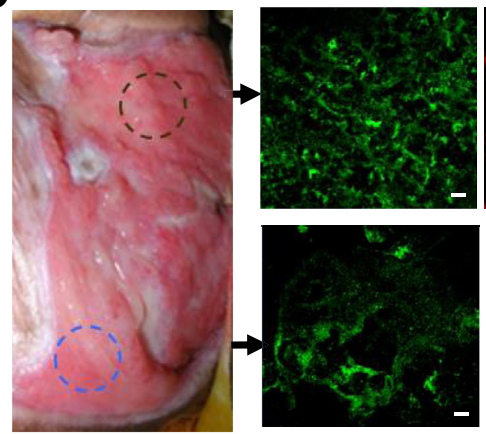

VG1
B

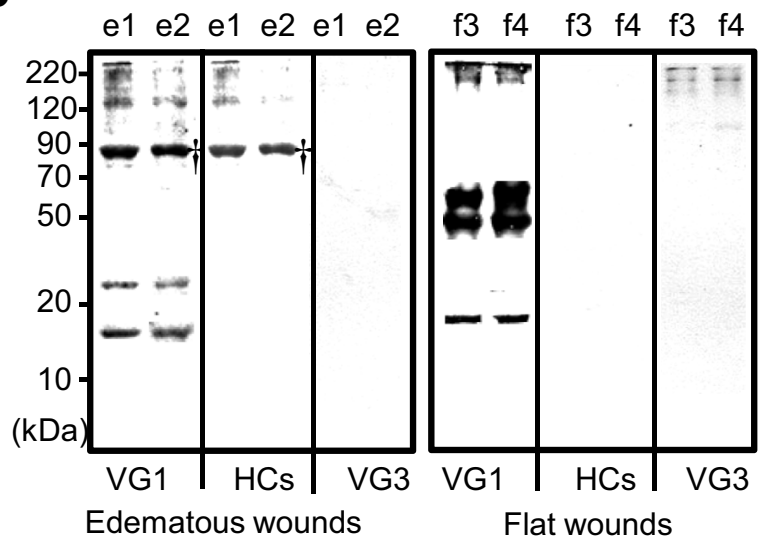

C

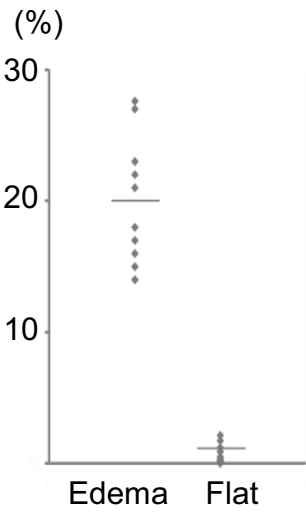

E
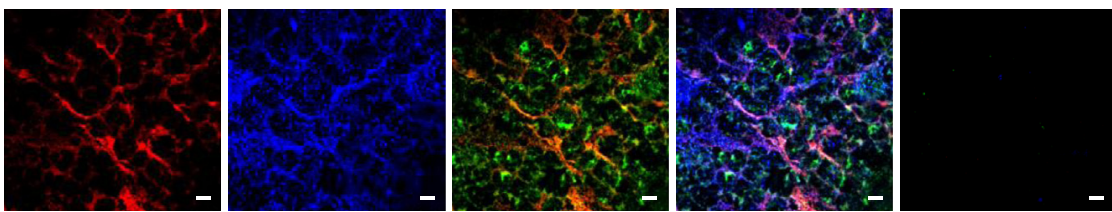

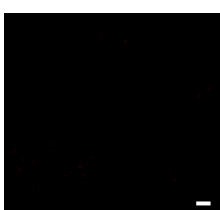

$\mathrm{HC} 1$

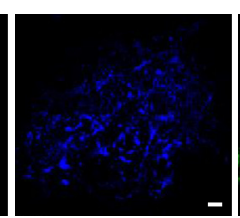

HA

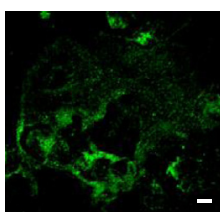

VG1/HC1

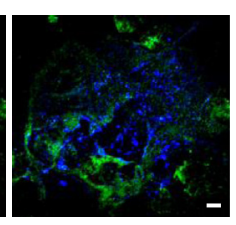

All

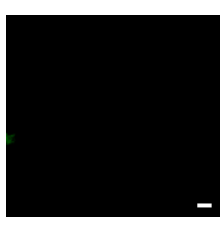

Control

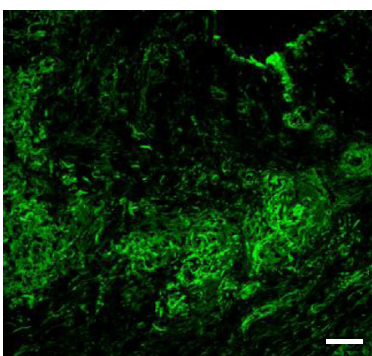

DPEAAE

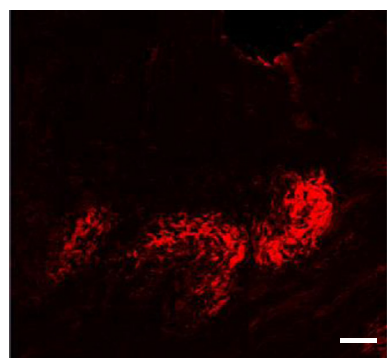

$\mathrm{HC}$

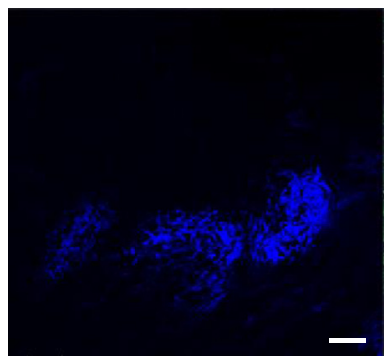

Macrophage

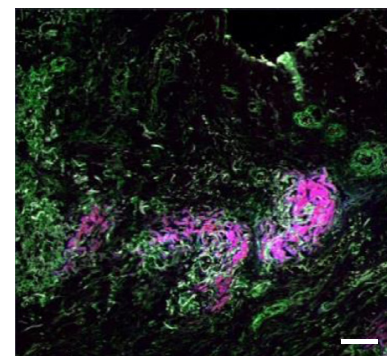

Merge

Figure 11 The versican G1 fragment (VG1F)-serum-derived hyaluronan (HA)-associated protein-HA complex in human pressure ulcers. A: In human pressure ulcers, edematous wounds (e1 and e2) typically become flat and stabilized ( $\mathrm{f} 3$ and $\mathrm{f} 4$ ) after appropriate treatments. B: The VG1F-HC complex was detected from the protein extract of wound surfaces shown in $\mathbf{A}$ (e1 and e2). The samples are indicated at the top of the figure and correspond to those shown in A. The samples were extracted with $6 \mathrm{~mol} / \mathrm{L} \mathrm{GdnHCl}$ and treated with trypsin and V8 protease (Materials and Methods); separated using SDS-PAGE under nonreducing conditions; and blotted. Incubation with the indicated antibodies revealed the cross-linked heavy chain (HC) and VG1 species (dagger). The bands of cross-linked HC and VG1 species (dagger) disappeared during wound healing. C: Paired samples from edematous or flat wounds were analyzed using Western blotting, and the bands shown in B were quantified. The relative intensities of the bands [VG1-HCs (daggers in B)]/the total polyclonal antibody 6084-positive bands (all VG1) differ significantly $(P=0.02)$ between edematous and flat wounds. D: Direct immunofluorescence of wound surface samples obtained from different sites of one individual wound. Samples obtained from the edematous wound (green dashed circle) and flat wound (blue dashed circle) were fixed and incubated with VG1 and HC1 antibodies, as well as biotin-conjugated HA-binding protein, as indicated. A representative result from triplicate experiments with different samples is shown. E: Immunohistochemical analysis of pressure ulcer tissue. The specimen was stained, as indicated. The merged images show VG1F colocalization, as detected by antibodies for DPEAAE epitope, HC1, and macrophage CD68. Data are expressed as means \pm SD (C). $n=10$ (C, each paired sample). Scale bars: $5 \mu \mathrm{m}$ (D); $100 \mu \mathrm{m}$ (E).

Merged images demonstrated that the colocalization of VG1F, HC, and HA become evident on rVN addition, suggesting that exogenous $\mathrm{rVN}$ enhances the VG1FSHAP-HA complex formation (Figure 7, E-G). Consistently, complex formation was not evident in the absence of rVN (Figure 7, M-O). The negative controls for primary probes are shown (Figure $7, \mathrm{H}$ and $\mathrm{P}$ ). These results indicated that addition of VG1F enhances the VG1FSHAP-HA complex deposition in the pericellular matrix produced by dermal fibroblasts. 


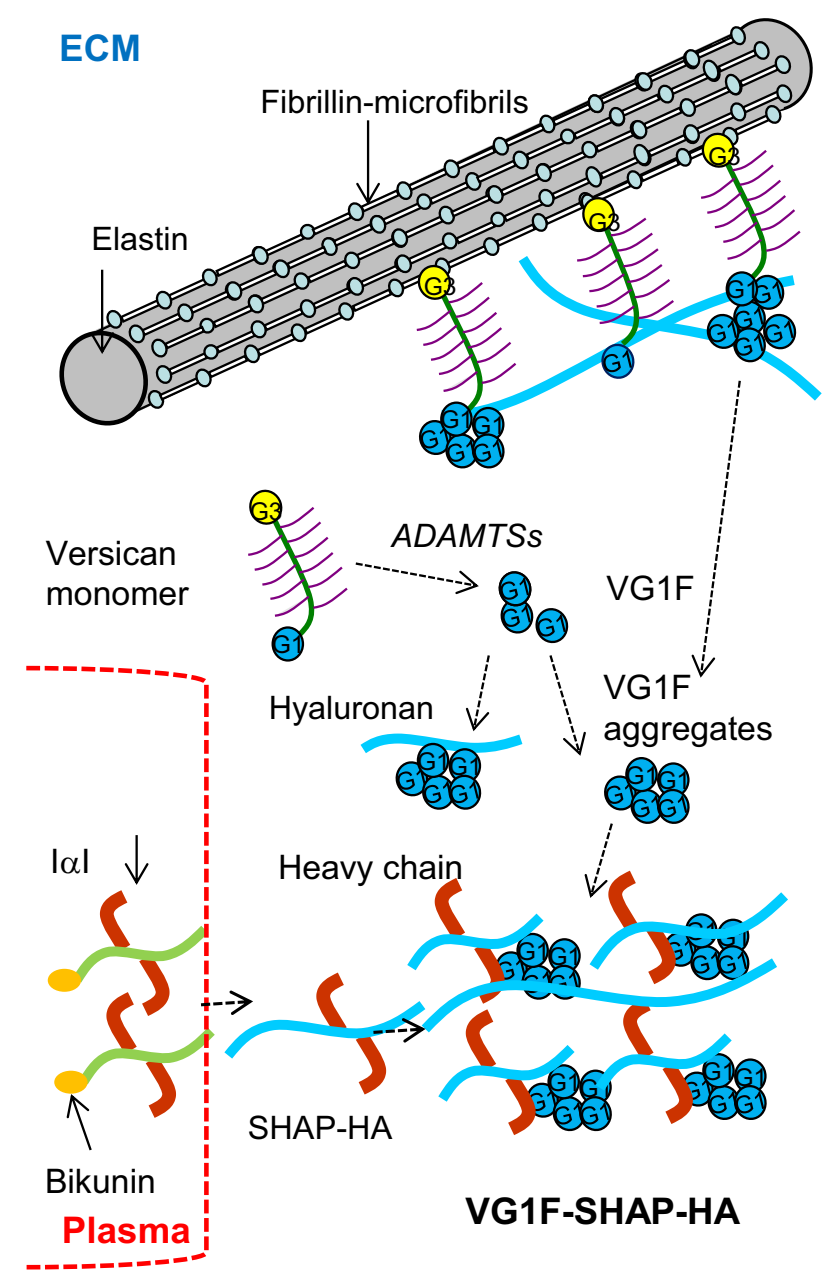

Figure 12 Proposed model of the versican $\mathrm{G} 1$ fragment-serum-derived hyaluronan-associated protein-hyaluronan (VG1F-SHAP-HA) complex formation in granulation tissue mediated via VG1F, HA, and inter- $\alpha$-trypsin inhibitor (I $\alpha \mathrm{I}$ ) interactions. Versicanase-type a disintegrin-like and metalloproteinase with thrombospondin motif (ADAMTS) proteases generate VG1F from versican-bound microfibrils or versican monomers. VG1F interacts homotypically and with heavy chain and HA. In wound tissues where serum and extracellular matrix (ECM) molecules interact, VG1F interacts with SHAP-HA. Consequently, the VG1F-SHAP-HA complex specifically forms in inflammatory environments, such as granulation tissue.

\section{Comparison of VG1F-SHAP-HA with Other VG1- and} HC-Containing Complexes

From the results described in the previous paragraph and our previous studies, ${ }^{13-15}$ versican, HCs, and HA were hypothesized to form distinct complexes in a tissue-specific manner. Thus, the constituents of the VG1F-SHAP-HA complex were compared with those of previously reported macrocomplexes. To this end, VG1F aggregates from the normal dermis, versican-bound microfibrils, intact versican monomers from conditioned medium, and rVN (recombinant VG1) were prepared according to our characterized procedures. ${ }^{10,14,32}$ As shown in the scheme (Figure 8), versican-bound microfibrils bound to anti-VG1, anti-VG3, anti-GAG $\alpha$, and anti-GAG $\beta$, but not to anti-DPEAAE antibody (Figure 1), suggesting that microfibril-bound versican was not cleaved within the GAG $\beta$ domain (Figure 8A). In contrast, the VG1F-SHAP-HA complex and VG1F aggregates from normal dermis were not detected by anti-GAG $\beta$, anti-VG3, or anti-fibrillin-1. Moreover, the VG1F-SHAP-HA complex reacted with anti-DPEAAE, suggesting the absence of the G3 domain attributable to ADAMTS-mediated cleavage within the versican GAG $\beta$ domain. In addition, the VG1F-SHAP-HA complex was detected by an A subdomain antibody (pAb 10693) (Supplemental Figure S1), indicating that cleavage between the $\mathrm{A}$ and $\mathrm{BB}^{\prime}$ subdomains does not occur in the formed complex. Furthermore, the complex was faintly positive to anti-GAG $\alpha$. On the basis of these results, it was concluded that the major versican species of the VG1F-SHAP-HA complex is VG1-GAG $\beta$-DPEAAE (Figure 1), although VG1-GAG $\alpha$-GAG $\beta$-DPEAAE is also present as a minor species.

The blot overlay assay showed that versican-bound microfibrils and VG1F-containing aggregates from normal dermis did not bind to bHABP, indicating that these aggregates did not contain HA (Figure 8A). However, VG1FSHAP-HA bound bHABP, indicating that HA is firmly linked to the complex, albeit through a noncovalent HAVG1 interaction. ${ }^{9}$ By dot blotting, $\mathrm{HC} 1$ was detected in the VG1F-SHAP-HA complex, but not in versican-bound microfibrils or VG1F aggregates from normal dermis.

Using similar methods, the VG1F-SHAP-HA complex was compared with I $\alpha$ I and SHAP-HA made by incubation of HA with fetal bovine serum. Both $\mathrm{HC} 1$ and $\mathrm{HC} 2$ were present in the VG1F-SHAP-HA complex and in SHAP-HA (Figure 8B). An anti-bikunin antibody did not detect SHAPHA and the VG1F-SHAP-HA complex (Figure 8B). ${ }^{25}$ In contrast, bikunin was present in $\mathrm{I} \alpha \mathrm{I}$ isolated from serum, suggesting that the VG1F-SHAP-HA complex is not a simple mixture of VG1F, I $\alpha$ I, and HA. ${ }^{26}$ In addition, the other HA-binding molecules, including TSG-6, HAPLN-1, and pentraxin 3, were not detected in the human and mouse VG1F-SHAP-HA complex, although TSG-6 was present in the serum (Supplemental Figure S3). This finding is consistent with the immunohistochemical analysis of wound tissues (Figure 3). On the basis of these results, a schematic representation of the VG1F-SHAP-HA complex was prepared (Figure 8C). The molecular complexes containing VG1, HA, and SHAP were indicated on the basis of these data and those from our previous studies. ${ }^{14,26,32}$

Temporal Formation of the VG1F-SHAP-HA Complex in the Moist Wound-Healing Model

It was next determined whether the complex was associated with the inflammatory conditions of pressure ulcers. The current therapeutic strategy for chronic wounds, including pressure ulcers, is based on the concept of moist wound healing ${ }^{36}$; thus, we investigated the functional significance of the VG1F-SHAP-HA complex in a mouse model of moist wound healing using topical ointment. In this model, 
the wound tissue was more edematous at day 3 than at days 1 and 6 (Figure 9A), suggesting that an inflammatory response culminates around day 3 . In addition, the granulation tissue was markedly thicker at day 3 (Figure 9A). Histological findings revealed marked infiltration of inflammatory cells within the edematous tissue at day 3, but not at days 1 and 6 (Figure 9B). Consistently, the VG1FSHAP-HA complex was transiently observed in the granulation tissues of day 2 (data not shown) and day 3 lesions (Figure 9C). In contrast, the VG1F-SHAP-HA complex was not apparent at days 1 and 6 , and the tissues were nonedematous (Figure 9, A-C). In addition, the VG1F-SHAPHA complex appeared to be associated with macrophage accumulation (Figure 9D). For further confirmation of complex formation, the wound tissue extract was sieved and used in dot blot and blot overlay analyses, which confirmed the presence of the VG1F-SHAP-HA complex in tissues from day 3, but not those from day 1 or 6 (Figure 10). However, the individual molecules were detected in the tissue at all stages (Figure 10).

Dot blot analyses on sieved fractions from a day 3 extract that was not concentrated by a cutoff filter revealed TSG-6 was not detected in the VG1F-SHAP-HA complex (Supplemental Figure S4A). Western blot analysis with multiple anti-TSG-6 antibodies revealed the monomeric form of TSG-6 in the granulation tissue (Supplemental Figure S4B). Further isolation using cesium chloride ultracentrifugation under dissociating conditions demonstrated the presence of the VG1F-SHAP-HA complex (Supplemental Figure S4C) not containing other HAbinding molecules (Supplemental Figure S3).

Presence of the VG1F-SHAP-HA Complex on the Wound Surfaces of Pressure Ulcers Showing an Inflammatory Appearance

To further study the role of the inflammatory response during wound healing, the VG1F-SHAP-HA complex was also examined using clinical samples from pressure ulcers (Figure 11A). Because conventional Western blotting did not resolve the VG1F-SHAP-HA complex, the HC-VG1F complex was detected from the wound surface by analyzing the fragments generated by enzymatic digestion.

Notably, fragments that reacted with HC and VG1 antibodies were detected on the edematous wound surface, showing a pale appearance (Figure 11, A and B), but not on flat nonedematous wound surfaces (Figure 11, A and B). Statistical analysis demonstrated that the ratio of the HC-VG1F complex/total VG1 was significantly different between inflamed and noninflamed granulation tissues (Figure 11C).

To confirm these data using a different method, wound smear experiments were performed by directly staining the surface proteins from a single wound. Interestingly, VG1F, $\mathrm{HCs}$, and HA localized to the fibril-like structure in the samples from edematous inflammatory wounds (Figure 11D); only VG1F and HA colocalized in a punctate appearance in flat wounds. Consistent with these results, localization of the VG1F-SHAP-HA complex was associated with macrophage accumulation (Figure 11E), suggesting that the VG1F-SHAP-HA complex is found specifically in inflammatory wounds.

\section{Discussion}

In the present study, we demonstrated that VG1F and SHAP-HA formed a macrocomplex that colocalized with macrophages in inflamed granulation tissue of pressure ulcers, suggesting that the complex might play an important role during the inflammatory process in these lesions. Immunohistochemical analyses indicated that the VG1FSHAP-HA complex localized to specific regions of pressure ulcer lesions, but not to the normal dermis. The complex formed through possible covalent cross-linking between VG1F and HCs, as evidenced by the fact that it was isolated under dissociating conditions. In particular, cesium chloride centrifugation enabled the separation of the VG1FSHAP-HA complex from other VG1F-containing aggregates from the normal dermis ${ }^{14}$ and versican-bound microfibrils. ${ }^{32}$ The VG1F-SHAP-HA complex included HA and $\mathrm{I} \alpha \mathrm{I} \mathrm{HCs}$, but not bikunin, suggesting that it contained SHAP-HA. ${ }^{15,26}$ The complex contained the entire VG1 domain, including the A subdomain that enhances the versican/HA interaction, ${ }^{39}$ and the major versican species was VG1-GAG $\beta$-DPEAAE. VG3 and most of the GAG $\beta$ domains were absent, suggesting cleavage by ADAMTStype versicanase.

Electron microscopic imaging of the isolated VG1FSHAP-HA complex was heterogeneous in size and demonstrated a globular morphology that resembled VG1Fcontaining aggregates in the normal dermis; however, the complex was larger and had a different electron density distribution. ${ }^{14}$ The ultrastructure observed in the present study was heterogeneous and seemed to be different from that of SHAP-HA complexes isolated from the synovial fluid of patients with rheumatoid arthritis. ${ }^{29}$ Resistance of the VG1F-SHAP-HA complex to proteolysis may be consistent with the fact that the complex was isolated from chronic inflammatory wounds, because chronic wounds have a prominent proteolytic environment. ${ }^{37,38}$ The resistance to proteolysis excluded proteomic analyses of the complex in this study.

Consistent with the absence of VG3, the VG1F-SHAPHA complex did not localize to the fibrillin microfibril network through a VG3-fibrillin-1 interaction, in contrast to previous studies. ${ }^{13,14}$ The marked difference of the VG1F-SHAP-HA complex from VG1F aggregates and versican-bound microfibrils suggested that the distribution of the HA network is likely tissue dependent. Indeed, HA localized to the fibrillin microfibril network in the mature 
dermis, where VG1F aggregates and versican-bound microfibrils predominate. ${ }^{10}$ The VG1F-SHAP-HA complex appeared independently of fibrillin microfibrils in the granulation tissue (Figure 11).

VG1F generated by the ADAMTS-mediated cleavage of the versican core protein plays critical roles in the development of the limbs, palate, and heart. ${ }^{22,23,40-42}$ Addition of VG1F to cultured fibroblasts alters the organization of HA and affects the cellular phenotype. ${ }^{43}$ In contrast, VG1F recognized by the anti-DPEAAE antibody is also present in the mature dermis. ${ }^{14,21}$ Although VG1F localizes to the normal dermis and granulation tissues, its tissue-specific interactions with $\mathrm{HCs}, \mathrm{HA}$, and fibrillin can distinctly regulate VG1F availability. On the basis of our data, the various phenotypes observed in versicanase-type ADAMTS-deficient ${ }^{22,23}$ and versican-deficient mice ${ }^{44,45}$ may be explained by the differential formation of VG1containing macrocomplexes.

In a blot overlay assay, aggregate formation was mediated by the specific interaction between the VG1 domain and the I $\alpha$ I heavy chains. This interaction may require ADAMTSinduced cleavage and VG1F homotypical interactions. Notably, the addition of VG1F to the fibroblast cultures enhanced the VG1F-SHAP-HA complex deposition into the pericellular ECM.

Because TSG-6 has an HA-binding motif ${ }^{9}$ and promotes SHAP-HA formation, ${ }^{46-48}$ localization of TSG-6 in the VG1F-SHAP-HA complex was examined in this study. Indeed, covalent binding of VG1F to SHAP-HA is a key characteristic of the complex found in granulation tissue. TSG-6 was not observed to be associated with SHAP-HA in these lesions and was instead localized around the VG1FSHAP-HA complexes. Although TSG-6 was not incorporated in the VG1F-SHAP-HA complexes isolated in this study, TSG-6 may promote the VG1F-SHAP-HA complex formation. ${ }^{49}$

The mechanism underlying the formation of the VG1FSHAP-HA complex was not fully clarified in the present study. However, the following conclusions may be drawn: VG1Fcontaining aggregates from granulation tissue included VG1F, but not VG3; and the aggregates contained HCs, but not bikunin, indicating the presence of the SHAP-HA complex. Although we had previously reported an interaction between full-length versican and $\mathrm{I} \alpha \mathrm{I},{ }^{15}$ a specific interaction between VG1F and HCs appears to be essential for VG1F-SHAP-HA complex formation. For these interactions to occur, I $\alpha \mathrm{I}$ may be supplied through serum and may be secreted from immature blood vessels observed in inflamed granulation tissue. VG1F may be derived from intact versican and/or versican-bound microfibrils by ADAMTS proteases. On the basis of the findings in this study and our previous study, ${ }^{14}$ we have generated a model for VG1F complex formation in granulation tissue (Figure 12).

The VG1F-SHAP-HA complex was present exclusively in the inflammatory phase of moist wound healing and colocalized with macrophages in both human and mouse granulation tissue. In contrast, VG1F stably incorporated in the fibrillin microfibrils by the homotypic interaction is not associated with inflammatory reactions. ${ }^{10,14}$ These data suggest that the unique complex may coordinately regulate inflammation during wound healing, although versican, $\mathrm{I} \alpha \mathrm{I}$, and HA were all present individually in the absence of inflammation. Furthermore, the VG1F-SHAP-HA complex may be present in other inflammatory conditions and may play a role in tissue remodeling.

Previous studies have reported the individual functions of versican, I $\alpha \mathrm{I}$, and HA in inflammatory conditions, including wound healing and cancer stroma. For instance, HA, I $\alpha$ I, and versican form cable-like structures in proximal tubular kidney epithelial cells, ${ }^{50}$ and similar SHAP-HA-containing structures are found in inflammatory bowel disease tissue. ${ }^{30}$ In cancer stroma, HA deficiency inhibits neovascularization and macrophage trafficking. ${ }^{51}$ Versican recruits macrophages through toll-like receptors in cancer tissues. ${ }^{52}$ Interestingly, various forms of HA may be generated through different mechanisms during proinflammatory and anti-inflammatory responses. ${ }^{53}$ Moreover, versican and/or SHAP-HA are temporarily present during the inflammatory response. ${ }^{54-57}$ However, the formation of molecular complexes containing versican and SHAP-HA was not fully characterized under these conditions. Nevertheless, the distinct formation of complexes containing versican, HCs, and HA found in our present and past studies clearly indicates tissue- or status-dependent generation of HA-rich ECM. $^{13,14,32}$

Although acute wound tissue generally expresses high levels of HA and chondroitin sulfate proteoglycan, ${ }^{5,8,55}$ it does not exhibit impaired wound healing. ${ }^{2,3}$ On the other hand, pressure ulcers, which are refractory and chronic wounds, exhibit various features, such as angiogenesis and edema in stromal tissues ${ }^{4}$ and enhanced turnover of HA. ${ }^{58}$ Interestingly, the wound smear experiment and mouse wound model demonstrated the presence of individual molecules, including HA and versican, in the wounds without an inflammatory response. However, the VG1FSHAP-HA complex was characteristically detected in the inflammatory stage of pressure ulcers. Because immobilized HC-HA has an anti-inflammatory effect, ${ }^{59}$ the VG1FSHAP-HA complex formation may modulate the function of SHAP-HA via immobilization within the ECM.

On the basis of these results, the VG1F-SHAP-HA complex may induce a specific response to inflammatory cells by forming a distinct environment within the ECM. The spatiotemporal formation of these complexes in specific regions and stages of pressure ulcer may be required to regulate tissue inflammation and remodeling. Furthermore, the VG1F-SHAP-HA complex dissipates with inflammatory subsidence, indicating that it may be essential for woundhealing progression. Comparative analysis with other versican- and SHAP-containing macrocomplexes will provide a better understanding of HA-rich matrix formation in stable and remodeling tissues. 


\section{Acknowledgments}

We thank Masahiro Saito (Tohoku University, Sendai, Japan) and Naoki Itano (Kyoto Sangyo University, Kyoto, Japan) for valuable discussions; Masaharu Mori (Okayama Prefectural University, Soja, Japan) for help with pathologic analyses; Lisheng Zhuo (Aichi Medical University) for the recombinant tumor necrosis factor-stimulated gene-6; Tamayuki Shinomura (Tokyo Medical and Dental University, Tokyo, Japan) for the anti-mouse GAG $\alpha$ domain antibody; and Dr. Lynn Sakai (Shriners Hospital, Portland, OR) for polyclonal antibody 9543.

Y.M. performed histochemical and biochemical experiments; Y.M. and M.Y. performed cell culture experiments; H.N. and K.W. expressed the recombinant proteins and generated the antibodies; M.Y., M.Z., and K.K. prepared the reagents used in immunohistochemical and biochemical experiments; E.K. and H.K. acquired the clinical and pathologic samples and evaluated clinical information; Y.M. and Z.I. designed the study; Y.M., S.F., and Z.I. wrote the article; all authors reviewed the results and approved the final version of the article.

\section{Supplemental Data}

Supplemental material for this article can be found at https://doi.org/10.1016/j.ajpath.2017.10.015.

\section{References}

1. Eming SA, Krieg T, Davidson JM: Inflammation in wound repair: molecular and cellular mechanisms. J Invest Dermatol 2007, 127: $514-525$

2. Moali C, Hulmes DJS: Extracellular and cell surface proteases in wound healing: new players are still emerging. Eur J Dermatol 2009, 19:552-564

3. Eming SA, Martin P, Tomic-Canic M: Wound repair and regeneration: mechanisms, signaling, and translation. Sci Transl Med 2014, 6: $1-16$

4. Vande Berg JS, Rudolph R: Pressure (decubitus) ulcer: variation in histopathology - a light and electron-microscope study. Hum Pathol 1995, 26:195-200

5. Yeo TK, Brown L, Dvorak HF: Alterations in proteoglycan synthesis common to healing wounds and tumors. Am J Pathol 1991, 138: $1437-1450$

6. Frenkel JS: The role of hyaluronan in wound healing. Int Wound J 2014, 11:159-163

7. Petrey AC, de la Motte CA: Hyaluronan, a crucial regulator of inflammation. Front Immunol 2014, 5:101

8. Oksala O, Salo T, Tammi R, Hakkinen L, Jalkanen M, Inki P, Larjava H: Expression of proteoglycans and hyaluronan during wound-healing. J Histochem Cytochem 1995, 43:125-135

9. Day AJ, Prestwich GD: Hyaluronan-binding proteins. J Biol Chem 2002, 277:4575-4579

10. Hasegawa K, Yoneda M, Kuwabara H, Miyaishi O, Itano N, Ohno A, Zako M, Isogai Z: Versican, a major hyaluronan-binding component in the dermis, loses its hyaluronan-binding ability in solar elastosis. J Invest Dermatol 2007, 127:1657-1663

11. Zimmermann DR, Dours-Zimmermann MT, Schubert M, BrucknerTuderman L: Versican is expressed in the proliferating zone in the epidermis and in association with the elastic network of the dermis. J Cell Biol 1994, 124:817-825

12. Bode-Lesniewska B, Dours-Zimmermann MT, Odermatt BF, Briner J, Heitz PU, Zimmermann DR: Distribution of the large aggregating proteoglycan versican in adult human tissues. J Histochem Cytochem 1996, 44:303-312

13. Isogai Z, Aspberg A, Keene DR, Ono RN, Reinhardt DP, Sakai LY: Versican interacts with fibrillin-1 and links extracellular microfibrils to other connective tissue networks. J Biol Chem 2002, 277: $4565-4572$

14. Murasawa Y, Watanabe K, Yoneda M, Zako M, Kimata K, Sakai LY, Isogai Z: Homotypic versican g1 domain interactions enhance hyaluronan incorporation into fibrillin microfibrils. J Biol Chem 2013, 288:29170-29181

15. Yoneda M, Zhao M, Zhuo LS, Watanabe H, Yamada Y, Huang L, Nagasawa S, Nishimura H, Shinomura T, Isogai Z, Kimata K: Roles of inter-alpha-trypsin inhibitor and hyaluronan-binding proteoglycans in hyaluronan-rich matrix formation. New Frontiers in Medical Sciences: Redefining Hyaluronan. Edited by Abatangelo G, Weigel PH. Amsterdam: Elsevier Science, 2001. pp. 21-30

16. Koyama H, Hibi T, Isogai Z, Yoneda M, Fujimori M, Amano J, Kawakubo M, Kannagi R, Kimata K, Taniguchi SI, Itano N: Hyperproduction of hyaluronan in Neu-induced mammary tumor accelerates angiogenesis through stromal cell recruitment: possible involvement of versican/PG-M. Am J Pathol 2007, 170: 1086-1099

17. Takahashi $\mathrm{Y}$, Kuwabara H, Yoneda M, Isogai Z, Tanigawa N, Shibayama Y: Versican G1 and G3 domains are upregulated and latent transforming growth factor-beta binding protein-4 is downregulated in breast cancer stroma. Breast Cancer 2012, 19:46-53

18. Apte SS: A disintegrin-like and metalloprotease (reprolysin-type) with thrombospondin type 1 motif (ADAMTS) superfamily: functions and mechanisms. J Biol Chem 2009, 284:31493-31497

19. Sorrell JM, Carrino DA, Baber MA, Caplan AI: Versican in human fetal skin development. Anat Embryol 1999, 199:45-56

20. Sandy JD, Westling J, Kenagy RD, Iruela-Arispe ML, Verscharen C, Rodriguez-Mazaneque JC, Zimmermann DR, Lemire JM, Fischer JW, Wight TN, Clowes AW: Versican V1 proteolysis in human aorta in vivo occurs at the Glu(441)-Ala(442) bond, a site that is cleaved by recombinant ADAMTS-1 and ADAMTS-4. J Biol Chem 2001, 276:13372-13378

21. Carrino DA, Calabro A, Darr AB, Dours-Zimmermann MT Sandy JD, Zimmermann DR, Sorrell JM, Hascall VC, Caplan AI Age-related differences in human skin proteoglycans. Glycobiology 2011, 21:257-268

22. McCulloch DR, Nelson CM, Dixon LJ, Silver DL, Wylie JD, Lindner V, Sasaki T, Cooley MA, Argraves WS, Apte SS: ADAMTS metalloproteases generate active versican fragments that regulate interdigital web regression. Dev Cell 2009, 17:687-698

23. Enomoto H, Nelson CM, Somerville RPT, Mielke K, Dixon LJ, Powell K, Apte SS: Cooperation of two ADAMTS metalloproteases in closure of the mouse palate identifies a requirement for versican proteolysis in regulating palatal mesenchyme proliferation. Development 2010, 137:4029-4038

24. Yoneda M, Suzuki S, Kimata K: Hyaluronic-acid associated with the surfaces of cultured fibroblasts is linked to a serum-derived $85-\mathrm{kda}$ protein. J Biol Chem 1990, 265:5247-5257

25. Huang L, Yoneda M, Kimata K: A serum-derived hyaluronan-associated protein (SHAP) is the heavy-chain of the inter-alpha-trypsin inhibitor. J Biol Chem 1993, 268:26725-26730

26. Zhuo LS, Hascall VC, Kimata K: Inter-alpha-trypsin inhibitor, a covalent protein-glycosaminoglycan-protein complex. J Biol Chem 2004, 279:38079-38082

27. Zhao M, Yoneda M, Ohashi Y, Kurono S, Iwata H, Ohnuki Y, Kimata K: Evidence for the covalent binding of SHAP, heavy-chains of inter-alpha-trypsin inhibitor, to hyaluronan. J Biol Chem 1995, 270:26657-26663 
28. Kida D, Yoneda M, Miyaura S, Ishimaru T, Yoshida Y, Ito T, Ishiguro N, Iwata $\mathrm{H}$, Kimata $\mathrm{K}$ : The SHAP-HA complex in sera from patients with rheumatoid arthritis and osteoarthritis. J Rheumatol 1999, 26:1230-1238

29. Yingsung W, Zhuo LS, Mörgelin M, Yoneda M, Kida D, Watanabe H, Ishiguro N, Iwata H, Kimata K: Molecular heterogeneity of the SHAP-hyaluronan complex: isolation and characterization of the complex in synovial fluid from patients with rheumatoid arthritis. J Biol Chem 2003, 278:32710-32718

30. de la Motte CA, Hascall VC, Drazba J, Bandyopadhyay SK, Strong SA: Mononuclear leukocytes bind to specific hyaluronan structures on colon mucosal smooth muscle cells treated with polyinosinic acid:polycytidylic acid: inter-alpha-trypsin inhibitor is crucial to structure and function. Am J Pathol 2003, 163: $121-133$

31. Eba H, Murasawa Y, Iohara K, Isogai Z, Nakamura H, Nakamura H, Nakashima M: The anti-inflammatory effects of matrix metalloproteinase- 3 on irreversible pulpitis of mature erupted teeth. PLoS One 2012, 7:e52523

32. Ohno-Jinno A, Isogai Z, Yoneda M, Kasai K, Miyaishi O, Inoue Y, Kataoka T, Zhao JS, Li H, Takeyama M, Keene DR, Sakai LY, Kimata K, Iwaki M, Zako M: Versican and fibrillin-1 form a major hyaluronan-binding complex in the ciliary body. Invest Ophthalmol Vis Sci 2008, 49:2870-2877

33. Matsumoto K, Kamiya N, Suwan K, Atsumi F, Shimizu K, Shinomura T, Yamada Y, Kimata K, Watanabe H: Identification and characterization of versican/PG-M aggregates in cartilage. J Biol Chem 2006, 281:18257-18263

34. Salier JP, Martin JP, Lambin P, McPhee H, Hochstrasser K: Purification of the human-serum inter-alpha-trypsin inhibitor by zinc chelate and hydrophobic interaction chromatographies. Anal Biochem 1980, 109:273-283

35. Isogai Z, Shinomura T, Yamakawa N, Takeuchi J, Tsuji T, Heinegård D, Kimata K: 2B1 antigen characteristically expressed on extracellular matrices of human malignant tumors is a large chondroitin sulfate proteoglycan, PG-M/versican. Cancer Res 1996, 56: 3902-3908

36. Korting HC, Schoellmann C, White RJ: Management of minor acute cutaneous wounds: importance of wound healing in a moist environment. J Eur Acad Dermatol Venereol 2011, 25:130-137

37. Lazaro JL, Izzo V, Meaume S, Davies AH, Lobmann R, Uccioli L: Elevated levels of matrix metalloproteinases and chronic wound healing: an updated review of clinical evidence. J Wound Care 2016, $25: 277-287$

38. Yager DR, Nwomeh BC: The proteolytic environment of chronic wounds. Wound Repair Regen 1999, 7:433-441

39. Matsumoto K, Shionyu M, Go M, Shimizu K, Shinomura T, Kimata K, Watanabe H: Distinct interaction of versican/PG-M with hyaluronan and link protein. J Biol Chem 2003, 278: 41205-41212

40. Kern CB, Twal WO, Mjaatvedt CH, Fairey SE, Toole BP, IruelaArispe ML, Argraves WS: Proteolytic cleavage of versican during cardiac cushion morphogenesis. Dev Dyn 2006, 235:2238-2247

41. Kern CB, Norris RA, Thompson RP, Argraves WS, Fairey SE, Reyes L, Hoffman S, Markwald RR, Mjaatvedt CH: Versican proteolysis mediates myocardial regression during outflow tract development. Dev Dyn 2007, 236:671-683

42. Kern CB, Wessels A, McGarity J, Dixon LJ, Alston E, Argraves WS, Geeting D, Nelson CM, Menick DR, Apte SS: Reduced versican cleavage due to Adamts9 haploinsufficiency is associated with cardiac and aortic anomalies. Matrix Biol 2010, 29:304-316

43. Merrilees MJ, Zuo N, Evanko SP, Day AJ, Wight TN: G1 domain of versican regulates hyaluronan organization and the phenotype of cultured human dermal fibroblasts. J Histochem Cytochem 2016, 64: 353-363

44. Mjaatvedt CH, Yamamura H, Capehart AA, Turner D, Markwald RR: The Cspg2 gene, disrupted in the hdf mutant, is required for right cardiac chamber and endocardial cushion formation. Dev Biol 1998, 202:56-66

45. Hatano S, Kimata K, Hiraiwa N, Kusakabe M, Isogai Z, Adachi E, Shinomura T, Watanabe H: Versican/PG-M is essential for ventricular septal formation subsequent to cardiac atrioventricular cushion development. Glycobiology 2012, 22:1268-1277

46. Baranova NS, Foulcer SJ, Briggs DC, Tilakaratna V, Enghild JJ, Milner CM, Day AJ, Richter RP: Inter-alpha-inhibitor impairs TSG 6-induced hyaluronan cross-linking. J Biol Chem 2013, 288: 29642-29653

47. Rugg MS, Willis AC, Mukhopadhyay D, Hascall VC, Fries E, Fülöp C, Milner CM, Day AJ: Characterization of complexes formed between TSG-6 and inter-alpha-inhibitor that act as intermediates in the covalent transfer of heavy chains onto hyaluronan. J Biol Chem 2005, 280:25674-25686

48. Lauer ME, Glant TT, Mikecz K, DeAngelis PL, Haller FM, Husni ME, Hascall VC, Calabro A: Irreversible heavy chain transfer to hyaluronan oligosaccharides by tumor necrosis factor-stimulated gene-6. J Biol Chem 2013, 288:205-214

49. Briggs DC, Birchenough HL, Ali T, Rugg MS, Waltho JP, Ievoli E, Jowitt TA, Enghild JJ, Richter RP, Salustri A, Milner CM, Day AJ: Metal ion-dependent heavy chain transfer activity of TSG-6 mediates assembly of the cumulus-oocyte matrix. J Biol Chem 2015, 290: 28708-28723

50. Selbi W, de la Motte C, Hascall VC, Day AJ, Bowen T, Phillips AO: Characterization of hyaluronan cable structure and function in renal proximal tubular epithelial cells. Kidney Int 2006, 70:1287-1295

51. Kobayashi N, Miyoshi S, Mikami T, Koyama H, Kitazawa M, Takeoka M, Sano K, Amano J, Isogai Z, Niida S, Oguri K, Okayama M, McDonald JA, Kimata K, Taniguchi SI, Itano N: Hyaluronan deficiency in tumor stroma impairs macrophage trafficking and tumor neovascularization. Cancer Res 2010, 70:7073-7083

52. Kim S, Takahashi H, Lin W-W, Descargues P, Grivennikov S, Kim Y, Luo J-L, Karin M: Carcinoma-produced factors activate myeloid cells through TLR2 to stimulate metastasis. Nature 2009, 457:102-106

53. Jiang D, Liang J, Noble PW: Hyaluronan as an immune regulator in human diseases. Physiol Rev 2011, 91:221-264

54. Evanko SP, Potter-Perigo S, Bollyky PL, Nepom GT, Wight TN: Hyaluronan and versican in the control of human T-lymphocyte adhesion and migration. Matrix Biol 2012, 31:90-100

55. Chang MY, Tanino Y, Vidova V, Kinsella MG, Chan CK, Johnson PY, Wight TN, Frevert CW: A rapid increase in macrophage-derived versican and hyaluronan in infectious lung disease. Matrix Biol 2014, 35:162-173

56. Frey H, Schroeder N, Manon-Jensen T, Iozzo RV, Schaefer L: Biological interplay between proteoglycans and their innate immune receptors in inflammation. FEBS J 2013, 280:2165-2179

57. Zhuo L, Kanamori A, Kannagi R, Itano N, Wu J, Hamaguchi M, Ishiguro N, Kimata K: SHAP potentiates the CD44-mediated leukocyte adhesion to the hyaluronan substratum. J Biol Chem 2006, 281:20303-20314

58. Dechert TA, Ducale AE, Ward SI, Yager DR: Hyaluronan in human acute and chronic dermal wounds. Wound Repair Regen 2006, 14: $252-258$

59. He H, Zhang SZ, Tighe S, Son J, Tseng SCG: Immobilized heavy chain-hyaluronic acid polarizes lipopolysaccharide-activated macrophages toward M2 phenotype. J Biol Chem 2013, 288:25792-25803 